\title{
THE NUCLEON "TENSOR CHARGES" AND THE SKYRME MODEL
}

by

\author{
James M. Olness
}

Submitted to the Department of Physics

in Partial Fulfillment of

the Requirements for the Degree of

Master of Science

at the

Massachusetts Institute of Technology

June 1994

(C) Massachusetts Institute of Technology 1994

All Rights Reserved

Signature of Author

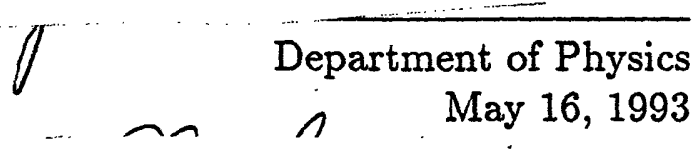

Certified by

Profeśsor Robert L. Jaffe Thesis Supervisor

Accepted by

MASSACHUSETTS INSTITUTE

OF TERHNDLDGY

MAY 251994

LIBRARIES

science 


\title{
THE NUCLEON "TENSOR CHARGES" AND THE SKYRME MODEL
}

\author{
by \\ James M. Olness \\ Submitted to the Department of Physics \\ on May 16, 1994 in partial fulfillment \\ of the requirements for the Degree of \\ Master of Science in Physics
}

\begin{abstract}
The lowest moment of the twist-two, chiral-odd parton distribution $h_{1}(x)$ of the nucleon can be related to the so-called "tensor charges" of the nucleon. We consider the tensor charges in the Skyrme model, and find that in the large- $N_{c}, \mathrm{SU}(3)$-symmetric limit, the model predicts that the octet isosinglet tensor charge, $g_{T}^{8}$, is of order $1 / N_{c}$ with respect to the octet isovector tensor charge, $g_{T}^{3}$. The predicted $F / D$ ratio is then $1 / 3$, in the large- $N_{c}$ limit. These predictions coincide with the Skyrme model predictions for the octet axial charges, $g_{A}^{8}$ and $g_{A}^{3}$. (The prediction $F / D=1 / 3$ for the axial charges differs from the commonly quoted prediction of $5 / 9$, which is based on an inconsistent treatment of the large- $N_{c}$ limit.) The model also predicts that the singlet tensor charge, $g_{T}^{0}$, is of order $1 / N_{c}$ with respect to $g_{T}^{3}$.
\end{abstract}

Thesis Supervisor: Dr. Robert L. Jaffe

Title: Professor of Physics 


\section{Acknowledgements}

I would like to thank my thesis supervisor, Professor Robert L. Jaffe, for his patience and help in completing this work. I would also like to express my heartfelt thanks to the many people who have helped me to keep my sanity while at M.I.T., especially Alexis and Betty, Ken, Oliver and Stan, Charlie, Frank, Yusuf, Mike, John, and Larry. And let's not forget Peggy! 


\section{Table of Contents}

1. Introduction

Page 5

2. Parton Distributions and $h_{1}(x)$

Page 7

3. The Skyrme Model

Page 22

4. Calculation of the Tensor Charges

page 39

Appendix: Anomalous Dimension of Tensor Charges

Page 51

Bibliography

Page 54 


\section{INTRODUCTION}

In certain types of high energy experiment involving nucleons, it is possible to parameterize the experimental cross section in terms of a collection of so-called 'parton distributions'. The parton distributions summarize those intrinsic properties of the nucleon which are most important in determining the experimental cross section. Not all parton distributions are of equal significance in these experiments. Indeed, a hierarchy can generally be established, whereby parton distributions are ranked according to their expected level of impact on the experimental cross section. This leads to the useful concept of 'twist'. Generally speaking, the parton distributions which are of greatest importance in these experiments are those of lowest possible twist; the contributions of higher-twist distributions are suppressed by one or more factors of some large momentum transfer, $Q$, characteristic of the process in question [1]. It is of interest, therefore, to identify and study the parton distributions of lowest twist.

The nucleon has three parton distributions at lowest twist, that is, twist-two. Two of these, $f_{1}(x)$ and $g_{1}(x)$, have been studied extensively, and have been measured in deep-inelastic scattering experiments [2]. The remaining twist-two distribution, $h_{1}(x)$, is relatively new, and has not been thoroughly studied [3]. It plays a negligible role in inclusive deep-inelastic lepton scattering experiments, so it has not yet been measured experimentally. In general, however, it is expected to contribute to hard processes at leading order in $1 / Q$ [4]. It can be shown, for example, that $h_{1}(x)$ plays an important role in polarized Drell-Yan processes [5].

The moments of $h_{1}(x)$ can be related to nucleon matrix elements of local operators. The low moments are especially interesting, because they determine the gross features 
of the distribution. In particular, the lowest moment of $h_{1}(x)$ can be related to the so-called tensor charge, $\delta q$ :

$$
\delta q=\int_{-1}^{1} d x h_{1}(x)
$$

Note that $h_{1}(x)$ carries flavor indices, which have been suppressed; there is an independent tensor charge, $\delta q$, for each quark flavor. The tensor charge derives its name from its relation to the following nucleon matrix element:

$$
\left\langle P S\left|\bar{q} \sigma_{\mu \nu} i \gamma^{5} q\right|_{Q^{2}} \mid P S\right\rangle=\frac{2}{M}\left(S_{\mu} P_{\nu}-S_{\nu} P_{\mu}\right) \delta q\left(Q^{2}\right)
$$

where $q=u, d, s$, etc. The parameter $Q^{2}$ appearing in equations (1) and (2) is a renormalization scale label, necessary to render $h_{1}(x)$ and the tensor charges well-defined in perturbative QCD. The tensor charges do not mix with other operators under renormalization, and are therefore characterized by a single anomalous dimension, which is calculated in the appendix. Henceforth, we will suppress all dependence on $Q^{2}$.

At present, it is not possible to calculate the tensor charges rigorously in $\mathrm{QCD}$. In order to study them, we must therefore turn to models. The purpose of this thesis is to describe the results of a Skyrme model analysis of the tensor charges. Section 2 presents a brief introduction to parton distributions, with emphasis on $h_{1}(x)$. Section 3 presents an overview of the Skyrme model. Finally, Section 4 presents the results of the analysis, along with thorough discussion. 


\section{PARTON DISTRIBUTIONS AND $h_{1}(x)$}

The easiest way to introduce the concept of a parton distribution is through the example of deep-inelastic scattering. Historically, this is where the concept originated, and it affords a particularly clean introduction to some of the basic ideas.

In a typical deep-inelastic scattering experiment, high energy leptons are used to probe a hadronic target, such as hydrogen or iron. The probe particles interact with the target by means of electroweak gauge bosons, which couple weakly to both target and probe. The scattering cross section can therefore be calculated approximately by keeping only the lowest order terms in the electroweak interactions. In electroproduction, for example, a representative amplitude is given by

$$
A \propto \bar{u}\left(l^{\prime}\right) \gamma_{\mu} u(l) \frac{1}{q^{2}}\left\langle X\left|J^{\mu}\right| P\right\rangle
$$

This is represented graphically in figure 2.1. The initial and final momenta of the electron are denoted by $l$ and $l^{\prime}$, respectively, while $q \equiv l-l^{\prime}$ is the photon momentum. The initial target state is denoted by $|P\rangle$, and the final hadronic state is denoted by $|X\rangle . J^{\mu}$ is the hadronic (quark) electromagnetic current operator. Typically, only the outgoing electron is observed, so the cross section involves a sum over all possible outgoing hadronic states:

$$
d \sigma \propto W_{\mu \nu} \equiv \frac{1}{4 \pi} \sum_{X}\left\langle p\left|J_{\mu}\right| X\right\rangle\left\langle X\left|J_{\nu}\right| p\right\rangle(2 \pi)^{4} \delta^{4}\left(P+q-P_{X}\right)
$$

By using translation operators and the completeness relation, we can put $W_{\mu \nu}$ in the form of an integral:

$$
W_{\mu \nu}=\frac{1}{4 \pi} \int d^{4} \xi e^{i q \cdot \xi}\left\langle P\left|J_{\mu}(\xi) J_{\nu}(0)\right| P\right\rangle
$$


The next step is to replace the product $J_{\mu}(\xi) J_{\nu}(0)$ with the commutator $\left[J_{\mu}(\xi), J_{\nu}(0)\right]$. (The new term vanishes for stable targets when $q^{0}$ is positive. To see this, simply reverse the steps that led from equation (2.2) to equation (2.3).) This is an important step, because it provides an opportunity to apply a bit of intuition.

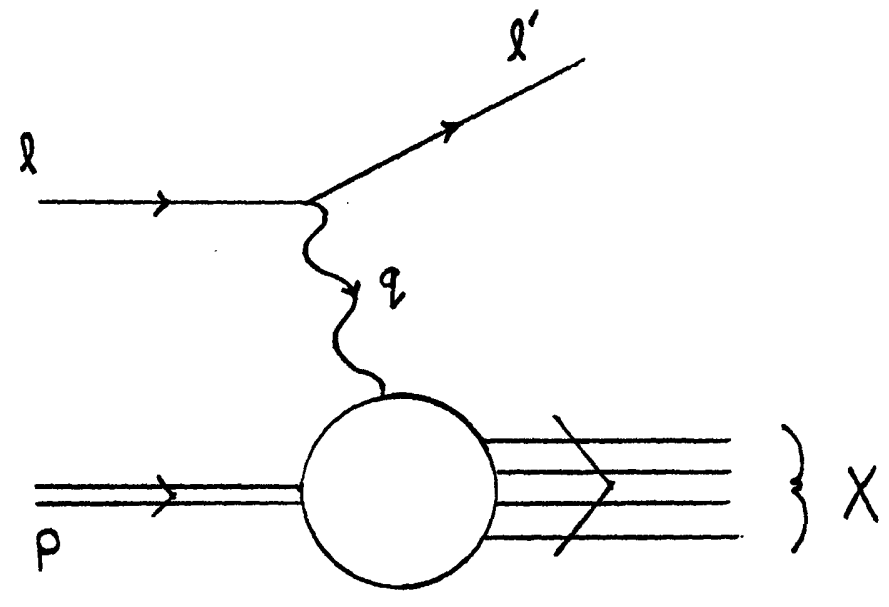

Fig. 2.1: Deep-inelastic electron scattering.

Causality requires that the current commutator vanish when $\xi$ is outside the lightcone. The commutator must therefore be singular when $\xi$ is on the light-cone. This singularity is easily demonstrated in free field theory. In the realistic case, when the quarks are interacting, we can imagine writing the commutator as an infinite series of terms in perturbation theory. The free field commutator then becomes the first term in this series. We will argue below that in a certain kinematic regime, known as the 'Bjorken limit', $W_{\mu \nu}$ is dominated by the light-cone singularity of the current commutator. If we can show, in addition, that the free field commutator represents the leading light-cone singularity, we will have achieved an important simplification.

Before proceeding, we will need to lay some groundwork. The hadronic tensor $W_{\mu \nu}$ is somewhat awkward to work with, because it carries lorentz indices. We need 
not carry around all sixteen components of $W_{\mu \nu}$, however, because it can be described completely in terms of two readily identifiable tensors. Using lorentz invariance, current conservation (i.e., $q^{\mu} W_{\mu \nu}=q^{\nu} W_{\mu \nu}=0$ ), and parity invariance (which rules out the tensor $\left.\epsilon_{\mu \nu \lambda \rho} P^{\lambda} q^{\rho}\right)$, it is easy to show that $W_{\mu \nu}$ can be written in the following form:

$$
W_{\mu \nu}=-\left(g_{\mu \nu}-\frac{q_{\mu} q_{\nu}}{q^{2}}\right) F_{1}\left(Q^{2}, \nu\right)+\left(P_{\mu}-\frac{P \cdot q}{q^{2}} q_{\mu}\right)\left(P_{\nu}-\frac{P \cdot q}{q^{2}} q_{\nu}\right) \frac{F_{2}\left(Q^{2}, \nu\right)}{\nu}
$$

where $Q^{2} \equiv-q^{2}$ and $\nu \equiv P \cdot q / M_{T}$. (We are assuming the target is unpolarized, so the spin vector, $S_{\mu}$, is not available for construction of tensors.) The scalar functions $F_{1}$ and $F_{2}$ are called 'structure functions'. The invariant mass (squared) of the final hadronic state is given by

$$
M_{X}^{2}=(P+q)^{2}=M_{T}^{2}+q^{2}+2 P \cdot q
$$

The requirement that $M_{X}$ be greater than or equal to the target mass, $M_{T}$, leads to the requirement that $x \equiv Q^{2} / 2 M_{T} \nu$ be less than or equal to unity. It is easy to show that $x$ must also be positive. $x$ is called the Bjorken scaling variable, and it has a special status in deep-inelastic scattering. The so-called 'deep-inelastic', or 'Bjorken' limit refers to the limit where $Q^{2}$ and $\nu$ are allowed to become large, while $x$ is kept fixed.

The Bjorken limit will be easier to grasp if we introduce a more natural set of coordinates. We will work in the rest frame of the target, and assume that in this frame, the photon travels in the $-\hat{z}$ direction. The photon four-momentum is then given by

$$
q=\left(\nu, 0,0,-\sqrt{\nu^{2}+Q^{2}}\right) .
$$

In the Bjorken limit, this becomes

$$
q \approx\left(\nu, 0,0,-\nu-M_{T} x\right)
$$


Now we introduce the so-called light-cone coordinates:

$$
q^{ \pm}=\frac{1}{\sqrt{2}}\left(q^{0} \pm q^{3}\right)
$$

For future reference, $q \cdot \xi=q^{+} \xi^{-}+q^{-} \xi^{+}-\mathbf{q}_{\perp} \cdot \xi_{\perp}$, and $\xi^{2}=2 \xi^{+} \xi^{-}-\xi_{\perp}^{2}$. In the Bjorken limit, $q^{-} \approx\left(2 \nu+M_{T} x\right) / \sqrt{2}$ and $q^{+} \approx-M_{T} x / \sqrt{2}$. Thus, $q^{-}$diverges in the Bjorken limit, while $q^{+}$approaches a fixed limit.

To proceed, it will be useful to understand how singularities in a function dominate its fourier transform when the transform variable becomes large. Consider a function $f\left(\xi^{+}, \xi^{-}\right)$which vanishes identically for $\xi^{+}<0$. (In the sense of analytic function theory, $f$ is singular at $\xi^{+}=0$. If we regard $\xi^{-}$as a fixed parameter and expand $f$ in a taylor series about $\xi^{+}=0$, the series will have zero radius of convergence.) The fourier transform of $f$ takes the form

$$
\begin{aligned}
\widetilde{f}\left(q^{-}, q^{+}\right) & =\int d \xi^{+} d \xi^{-} e^{i\left(q^{-} \xi^{+}+q^{+} \xi^{-}\right)} f\left(\xi^{+}, \xi^{-}\right) \\
& =\int_{0}^{\infty} d \xi^{+} e^{i q^{-} \xi^{+}} \int_{-\infty}^{\infty} d \xi^{-} e^{i q^{+} \xi^{-}} f\left(\xi^{+}, \xi^{-}\right)
\end{aligned}
$$

Repeated integration by parts in the $\xi^{+}$variable can be used to put this in the form of a series in inverse powers of $q^{-}$:

$$
\widetilde{f}\left(q^{-}, q^{+}\right)=\frac{i}{q^{-}} f_{1}\left(q^{+}\right)+\left(\frac{i}{q^{-}}\right)^{2} f_{2}\left(q^{+}\right)+\ldots
$$

where

$$
\begin{aligned}
& f_{1}\left(q^{+}\right) \equiv \int d \xi^{-} e^{i q^{+} \xi^{-}} f\left(0, \xi^{-}\right), \\
& \left.f_{2}\left(q^{+}\right) \equiv \int d \xi^{-} e^{i q^{+} \xi^{-}} \frac{\partial f\left(\xi^{+}, \xi^{-}\right)}{\partial \xi^{+}}\right|_{\xi^{+}=0}
\end{aligned}
$$

Thus, when $q^{-}$becomes large, the fourier transform is determined by the behavior of $f$ in the neighborhood of its singularity at $\xi^{+}=0$. 
The significance of the Bjorken limit can now be understood by observing that the current commutator $\left[J_{\mu}(\xi), J_{\nu}(0)\right]$ is singular when $\xi^{+}=0$ (and $\left.\xi_{\perp}=0\right)$. Since the conjugate transform variable, $q^{-}$, diverges in the Bjorken limit, $W_{\mu \nu}$ will be dominated by the behavior of $\left[J_{\mu}(\xi), J_{\nu}(0)\right]$ in the region $\xi^{+} \approx 0$. It can be shown that the corresponding domain for $\xi^{-}$satisfies $\left|\xi^{-}\right| \lesssim 1 / q^{+} \approx \sqrt{2} / M_{T} x$ [6]. (This is basically an uncertainty principle argument: $\Delta \xi^{-} \Delta q^{+} \sim 1$.) Thus, in the Bjorken limit, $W_{\mu \nu}$ is dominated by the behavior of the current commutator in the region $\xi^{+} \xi^{-} \approx 0$. Since the commutator vanishes when $\xi^{2}=2 \xi^{+} \xi^{-}-\xi_{\perp}^{2}$ is negative, we see that the region of interest is in fact the neighborhood of the light-cone, $\xi^{2} \approx 0$.

We have made an important first step by identifying the light-cone singularity of $\left[J_{\mu}(\xi), J_{\nu}(0)\right]$ as the primary determinant of $W_{\mu \nu}$ (in the Bjorken limit). The next step is to isolate the leading singularity of the commutator. In the language of the simple example above, we would like to find a function $f_{1}\left(q^{+}\right)$which represents the dominant contributions to $W_{\mu \nu}$ when $q^{-}$becomes large (i.e., in the Bjorken limit).

We would like to study the various terms in the perturbative expansion of the current commutator, $\left[J_{\mu}(\xi), J_{\nu}(0)\right]$. Generally speaking, time-ordered products are easier to handle than commutators, because we can use ordinary Feynman diagrams. Therefore, we consider the forward virtual compton scattering amplitude:

$$
T_{\mu \nu}=i \int d^{4} \xi e^{i q \cdot \xi}\left\langle p\left|T\left(J_{\mu}(\xi) J_{\nu}(0)\right)\right| p\right\rangle
$$

This is represented graphically in figure 2.2. It is straightforward to show that $T_{\mu \nu}$ and $W_{\mu \nu}$ are related:

$$
2 \pi W_{\mu \nu}=\operatorname{Im}\left(T_{\mu \nu}\right)
$$


(This is the optical theorem.) Figure 2.3 displays several low order graphs in the perturbative expansion of $T_{\mu \nu}$. The contributions of these graphs to $W_{\mu \nu}$ can be analyzed by means of the operator product expansion and dispersion theory techniques [7]. Using

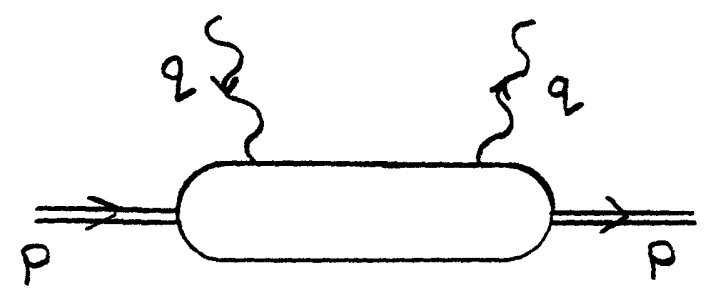

Fig. 2.2: Forward virtual Compton scattering.

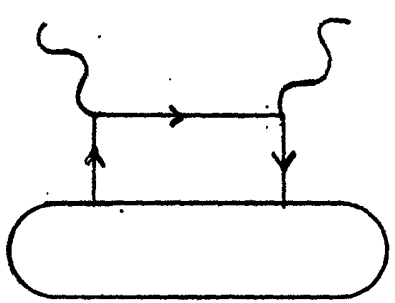

(a)

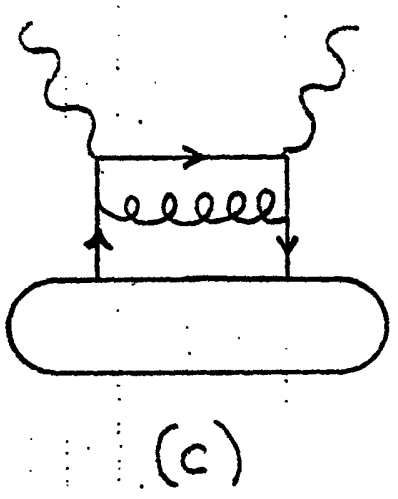

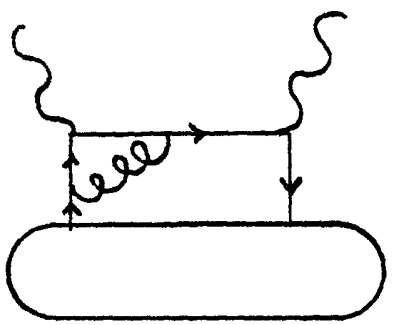

(b)

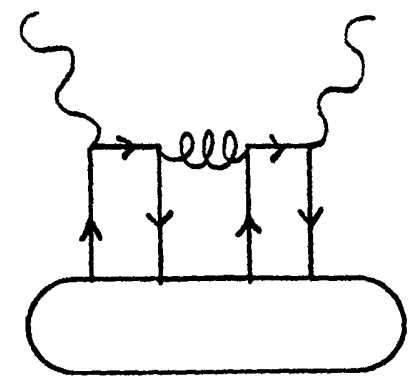

(d)

Fig. 2.3: Contributions to forward virtual Compton scattering amplitude. 
these methods, it can be shown that in the Bjorken limit, the so-called 'handbag diagram' in figure 2.3a represents the dominant contributions to $W_{\mu \nu}[8]$. The contributions of diagrams like the one in figure $2.3 \mathrm{~d}$ can be shown to be suppressed by factors of $1 / Q^{2}$, while diagrams like those in figures $2.3 \mathrm{~b}$ and $2.3 \mathrm{c}$ essentially renormalize the leading order diagram. I will have more to say about this shortly. Our goal is to isolate the leading contribution to $W_{\mu \nu}$, so henceforth, I will concentrate on figure 2.3a.

The diagram in figure 2.3a depicts what is essentially the elastic scattering of a free quark. Consequently, the imaginary part of this diagram is obtained by evaluating the current commutator $\left[J_{\mu}(\xi), J_{\nu}(0)\right]$ in free field theory. (This is just the optical theorem for scattering from a free quark.) The current operator, $J_{\mu}(\xi)$, is given by $\bar{\psi}(\xi) \gamma_{\mu} Q \psi(\xi)$, where $Q$ is the quark charge matrix: $Q=\operatorname{diag}(2 / 3,-1 / 3,-1 / 3, \ldots)$. The free field commutator is then given by

$$
\left[J_{\mu}(\xi), J_{\nu}(0)\right]=\bar{\psi}(\xi) \gamma_{\mu} S(\xi) \gamma_{\nu} Q^{2} \psi(0)-\bar{\psi}(0) \gamma_{\nu} S(-\xi) \gamma_{\mu} Q^{2} \psi(\xi)
$$

where $S(\xi)$ is the anticommutator function

$$
S(\xi) \equiv\{\psi(\xi), \bar{\psi}(0)\}=-\gamma^{\mu} \frac{\partial}{\partial \xi^{\mu}} \Delta(\xi)
$$

and

$$
\Delta(\xi)=\frac{1}{2 \pi} \delta\left(\xi^{2}\right) \epsilon\left(\xi^{0}\right)
$$

Terms involving quark masses have been neglected. As claimed earlier, the free field commutator is indeed singular at $\xi^{2}=0$. We have argued that in the Bjorken limit, this term dominates the scattering cross section. In fact, it also yields contributions to $W_{\mu \nu}$ which are suppressed by one or more factors of $1 / Q^{2}$, relative to the leading 
term. We would like to eliminate these terms, and isolate the dominant one. Reference to the simple example involving the two-dimensional fourier transform, equation (2.10), suggests that in the Bjorken limit (i.e., $q^{-} \rightarrow \infty$ ), we should be able to develop $W_{\mu \nu}$ in a series in inverse powers of $q^{-}$. The coefficients in this series should be given by fourier transforms of functions evaluated at $\xi^{+}=0$, or in other words, on the light-cone. This can indeed be done. I will not carry out the analysis here-it is a straightforward exercise in fourier analysis, the details of which can be found the Los Alamos lectures by Jaffe [9]. In terms of the structure functions defined earlier, the leading term in $1 / q^{-}$is given by

$$
F_{1}\left(Q^{2}, \nu\right)=\frac{1}{2} \sum e_{q}^{2}\left[f_{1}^{q}(x)+f_{1}^{\bar{q}}(x)\right]
$$

where $e_{q}$ is the electric charge of quark $q$, and

$$
\begin{aligned}
f_{1}^{q}(x) & =\frac{1}{4 \pi} \int e^{i q^{+} \xi^{-}}\left\langle P\left|\bar{q}\left(\xi^{-}\right) \gamma^{+} q(0)\right| P\right\rangle \\
f_{1}^{\bar{q}}(x) & =-\frac{1}{4 \pi} \int e^{i q^{+} \xi^{-}}\left\langle P\left|\bar{q}(0) \gamma^{+} q\left(\xi^{-}\right)\right| P\right\rangle=-f_{1}^{q}(-x)
\end{aligned}
$$

The light-cone gamma matrices $\gamma^{ \pm}$are defined in analogy with $q^{ \pm}: \gamma^{ \pm} \equiv\left(\gamma^{0} \pm \gamma^{3}\right) / \sqrt{2}$. The other structure function, $F_{2}\left(Q^{2}, \nu\right)$, is given by $F_{2}=2 x F_{1}$. The function $f_{1}(x)$ is referred to as a parton distribution. In the Bjorken limit, it represents the dominant contributions to $W_{\mu \nu}$, and can thus be thought of as summarizing those intrinsic properties of the target which are most important in determining the experimental cross section.

It is useful to point out explicitly the close analogy between the two-dimensional example, equation (2.10), and the present case of deep-inelastic scattering. When $q^{-}$ becomes large, the function $\widetilde{f}\left(q^{-}, q^{+}\right)$satisfies $\tilde{f} \approx \frac{i}{q^{-}} f_{1}\left(q^{+}\right)$. The dependence on $q^{-}$ becomes essentially trivial, while the dependence on $q^{+}$is given by a fourier transform 
over the region $\xi^{+}=0$. In the case of deep-inelastic scattering, the structure functions $F_{1}$ and $F_{2}$ become functions of $q^{+} \propto x$ in the Bjorken limit; the dependence on $q^{-}$ drops out (except for renormalization effects). The dependence on $x$ is given by a fourier transform over the region $\xi^{+}=\xi_{\perp}=0$. (Since the commutator $\left[J_{\mu}(\xi), J_{\nu}(0)\right]$ vanishes for $\xi^{2}=2 \xi^{+} \xi^{-}-\xi_{\perp}^{2}<0, \xi^{+}=0$ requires $\xi_{\perp}=0$ as well.)

As mentioned earlier, the simple 'handbag' diagram (fig. 2.3a) is not the only one which survives in the Bjorken limit. Graphs like those in figures $2.3 \mathrm{~b}$ and $2.3 \mathrm{c}$ also contribute at leading order in $1 / Q^{2}$ to the deep-inelastic scattering cross section. Their most important effect is to modify the simple scaling property exhibited by the free field term in $W_{\mu \nu}$ [8]. In free field theory, $F_{1}$ and $F_{2}$ are functions of $x$ alone, but in QCD, they also depend weakly (in the Bjorken limit) on $Q^{2}$. This dependence appears logarithmically, and can be understood as a consequence of the need to renormalize $f_{1}(x)$. Thus far, we have suppressed all dependence on $Q^{2}$, and we will continue to do so. It should be remembered, however, that $f_{1}(x)$ in fact depends weakly on $Q^{2}$.

The function $f_{1}(x)$ can be interpreted as a probability distribution (hence the name 'parton distribution'). To bring out this interpretation, we will need to lay some groundwork. First, we define the projection matrices, $P^{ \pm} \equiv \frac{1}{2} \gamma^{\mp} \gamma^{ \pm}$. It is straightforward to show that these have the properties required of projection matrices (i.e., $\left.P^{ \pm} P^{ \pm}=P^{ \pm}, P^{ \pm} P^{\mp}=P^{\mp} P^{ \pm}=0, P^{+}+P^{-}=1\right)$. We then define the ' ${ }^{\prime}$ ' and ' components of the quark field:

$$
\psi_{ \pm} \equiv P^{ \pm} \psi
$$

The function $f_{1}(x)$ can now be written as follows:

$$
f_{1}(x)=\frac{1}{2 \sqrt{2} \pi} \int d \xi^{-} e^{i q^{+} \xi^{-}}\left\langle P\left|\psi_{+}^{\dagger}\left(\xi^{-}\right) \psi_{+}(0)\right| P\right\rangle .
$$


If we insert a complete set of states and translate out the $\xi^{-}$dependence, we obtain

$$
f_{1}(x)=\frac{1}{\sqrt{2}} \sum_{n} \delta\left(P^{+}-x P^{+}-P_{n}^{+}\right)\left|\left\langle n\left|\psi_{+}\right| P\right\rangle\right|^{2} .
$$

In this form, $f_{1}(x)$ can be interpreted as the probability (per unit $x$ ) to remove from the target a quark with 'momentum' fraction $x$, leaving behind a physical state with $P_{n}^{+}=(1-x) P^{+}$. Using equation $(2.20)$, it is easy to show that $f_{1}^{q}(x)$ is properly normalized, in the sense that integration over all values of $x$ yields the number of quarks of type $q$. (There is a subtlety here: The distribution function does not vanish outside the physical region. The intermediate state $|n\rangle$ in equation (2.21) is a physical state, so $P_{n}^{+}$must be nonnegative, i.e., $E_{n} \geq\left|\mathbf{P}_{n}\right|$. This implies that $f_{1}(x)$ is zero when $x>1$. However, for $x<0, f_{1}(x)$ does not vanish, and in fact becomes negative; see equation (2.18). This issue is discussed extensively in the article by Jaffe [10]. We will simply ignore this subtlety, and restrict our integration to the physical region, $0<x<1$.) Remembering to subtract out the antiquarks, the (net) number of quarks of type $q$ should be given by

$$
\begin{aligned}
N_{q} & =\int_{0}^{1} d x\left[f_{1}^{q}(x)-f_{1}^{\bar{q}}(x)\right] \\
& =\int_{-\infty}^{\infty} d x f_{1}^{q}(x) .
\end{aligned}
$$

This integral is easily evaluated, using the fact that $\bar{\psi} \gamma_{\mu} \psi$ is a conserved current. Working in the rest frame of the target, we obtain

$$
\begin{aligned}
N_{q} & =\int d x \frac{1}{4 \pi} \int d \xi^{-} e^{i q^{+} \xi^{-}}\left\langle p\left|\bar{q}\left(\xi^{-}\right) \gamma^{+} q(0)\right| p\right\rangle \\
& =\frac{1}{2 M_{T}}\left\langle p\left|\bar{q}(0) \gamma^{0} q(0)\right| p\right\rangle
\end{aligned}
$$

which of course is an identity. 
The preceding remarks have been made with unpolarized targets in mind. When the target is polarized, new structure functions appear in $W_{\mu \nu}[2]$ :

$$
W_{\mu \nu} \equiv W_{\mu \nu}^{(S)}+W_{\mu \nu}^{(A)}
$$

where $W_{\mu \nu}^{(S)}$ is the symmetric part of $W_{\mu \nu}$, given in equation (2.4), and $W_{\mu \nu}^{(A)}$ is the antisymmetric part, given by

$$
W_{\mu \nu}^{(A)}=-i \epsilon_{\mu \nu \lambda \sigma} q^{\lambda}\left(\frac{S^{\sigma}}{\nu}\left(g_{1}\left(Q^{2}, \nu\right)+g_{2}\left(Q^{2}, \nu\right)\right)-(q \cdot S) P^{\sigma} \frac{g_{2}\left(Q^{2}, \nu\right)}{\nu^{2}}\right)
$$

The new structure functions can be measured by performing scattering experiments with longitudinally polarized leptons. In the Bjorken limit, methods similar to those described above can be used to write $g_{1}\left(Q^{2}, \nu\right)$ in terms of a new, polarized parton distribution, $g_{1}(x):$

$$
g_{1}(x)=\frac{1}{P^{+} \sqrt{2}} \int \frac{d \lambda}{2 \pi} e^{i \lambda x}\left\langle P S_{z}\left|\psi_{+}^{\dagger}(0) \gamma^{5} \psi_{+}(\lambda n)\right| P S_{z}\right\rangle,
$$

where $S_{z}$ indicates that the target is polarized longitudinally with respect to the beam. $n$ is a light-like vector with $n^{+}=\mathbf{n}_{\perp}=0$. It is easy to show that $f_{1}(x)$ can also be put in this general form.

As with $f_{1}(x), g_{1}(x)$ has a probabilistic interpretation. To demonstrate this, we introduce the chirality projection operators, $\chi^{ \pm} \equiv\left(1 \pm \gamma^{5}\right) / 2$, and define the right- and left-handed components of the quark operators:

$$
\begin{aligned}
& \psi_{+R} \equiv \chi_{+} \psi_{+} \\
& \psi_{+L} \equiv \chi-\psi_{+}
\end{aligned}
$$

Note that the light-cone projection operators commute with the chirality projection operators, so there is no ambiguity in these definitions. We can now write

$$
g_{1}(x)=\frac{1}{P+\sqrt{2}} \int \frac{d \lambda}{2 \pi} e^{i \lambda x}\left\langle P S_{z}\left|\psi_{+R}^{\dagger}(0) \psi_{+R}(\lambda n)-\psi_{+L}^{\dagger}(0) \psi_{+L}(\lambda n)\right| P S_{z}\right\rangle
$$


Thus, $g_{1}(x)$ can be interpreted as counting right-handed helicity quarks minus lefthanded helicity quarks. (Using the same notation, $f_{1}(x)$ is given by

$$
f_{1}(x)=\frac{1}{P^{+} \sqrt{2}} \int \frac{d \lambda}{2 \pi} e^{i \lambda x}\left\langle P S_{z}\left|\psi_{+R}^{\dagger}(0) \psi_{+R}(\lambda n)+\psi_{+L}^{\dagger}(0) \psi_{+L}(\lambda n)\right| P S_{z}\right\rangle
$$

Thus, $f_{1}(x)$ counts all quarks equally, regardless of polarization.)

At this point, it becomes appropriate to ask if there are other parton distributions that contribute to hard processes at leading order in $1 / Q^{2}$. In fact, there is one other such distribution. This is defined, in analogy with $f_{1}(x)$ and $g_{1}(x)$, as a light-cone fourier transform of a quark-quark correlation function, this time, in a transversely polarized target. The so-called 'transversity distribution', $h_{1}(x)$, is given by

$$
h_{1}(x)=\frac{1}{P^{+} \sqrt{2}} \int \frac{d \lambda}{2 \pi} e^{i \lambda x}\left\langle P S_{\perp}\left|\psi_{+}^{\dagger}(0) \gamma_{\perp} \gamma^{5} \psi_{+}(\lambda n)\right| P S_{\perp}\right\rangle
$$

where $S_{\perp}$ indicates that the target is polarized transversely with respect to the beam [3]. The requirement that the target be transversely polarized can be understood by writing $h_{1}(x)$ in terms of objects with more familiar lorentz transformation properties. It is easy to establish the following identity:

$$
\psi_{+}^{\dagger}(0) \gamma_{\perp} \gamma^{5} \psi_{+}(\lambda n)=\bar{\psi}(0)\left[\gamma^{+}, \gamma_{\perp}\right] \gamma^{5} \psi(\lambda n)
$$

Thus, the matrix element appearing in the definition of $h_{1}(x)$ is one component of a two index, antisymmetric pseudotensor. We can construct three such tensors from $P, S$ and $n: P_{\mu} S_{\nu}-P_{\nu} S_{\mu}, n_{\mu} S_{\nu}-n_{\nu} S_{\mu}$, and $\left(P_{\mu} n_{\nu}-P_{\nu} n_{\mu}\right) n \cdot S$. For $\mu={ }^{+}+$', and $\nu=$ ' $\perp$ ', only the first tensor is nonvanishing (recall $n^{+}=\mathbf{n}_{\perp}=0$ ). Since $\mathbf{P}_{\perp}=0$, the spin vector, $S_{\mu}$, must have a nonvanishing ' $\perp$ ' component in order that the matrix element 
be nonvanishing. (The requirement that the target be completely transversely polarized is simply a matter of definition.)

The distributions $f_{1}(x), g_{1}(x)$, and $h_{1}(x)$ are all defined in terms of the ' + ' components of the quark fields. This can be understood by using so-called 'light-cone quantization' techniques. A discussion of this, however, is beyond the scope of this thesis. The interested reader is referred to the article by Jaffe and $\mathrm{Ji}$ for details and references [1]. Using the generic form $\psi_{+}^{\dagger} \Gamma \psi_{+}$, with $\Gamma^{\dagger}=\Gamma$ (so the distribution is real), there can be no more than four distributions. This is because $\psi_{+}$has only two independent components, and the space of two-by-two hermitian matrices is four-dimensional. The matrices $1, \gamma^{5}, \gamma^{1} \gamma^{5}$, and $\gamma^{2} \gamma^{5}$ are a complete set of independent hermitian matrices on the two-dimensional space inhabited by $\psi_{+}$. We have defined distributions corresponding to three of these matrices. The fourth leads to a function which vanishes identically.

As with $f_{1}(x)$ and $g_{1}(x), h_{1}(x)$ has a probabilistic interpretation. This interpretation is obscured, however, in the chirality basis:

$$
h_{1}(x)=\frac{1}{P+\sqrt{2}} \int \frac{d \lambda}{2 \pi} e^{i \lambda x}\left\langle P S_{\perp}\left|\psi_{+L}^{\dagger}(0) \gamma_{\perp} \psi_{+R}(\lambda n)-\psi_{+R}^{\dagger}(0) \gamma_{\perp} \psi_{+L}(\lambda n)\right| P S_{\perp}\right\rangle .
$$

Evidently, $h_{1}(x)$ involves a change of chirality. In order to bring out the probabilistic interpretation of $h_{1}(x)$ we will need to define a new set of projection matrices. For notational convenience, I will temporarily assume the target is polarized in the $+\hat{x}$ direction. Then we define

$$
Q_{ \pm} \equiv \frac{1}{2}\left(1 \pm \gamma^{1} \gamma^{5}\right)
$$

It is easy to check that these projection matrices commute with the light-cone projection matrices, $P^{+}$and $P^{-}$. If we define

$$
\begin{aligned}
& \psi_{+\uparrow} \equiv Q_{+} \psi_{+} \\
& \psi_{+\downarrow} \equiv Q_{-} \psi_{+}
\end{aligned}
$$


then it is straightforward to show that $h_{1}(x)$ can be written in the form

$$
h_{1}(x)=\frac{1}{P^{+} \sqrt{2}} \int \frac{d \lambda}{2 \pi} e^{i \lambda x}\left\langle P S_{\perp}\left|\psi_{+\uparrow}^{\dagger}(0) \psi_{+\uparrow}(\lambda n)-\psi_{+\downarrow}^{\dagger}(0) \psi_{+\downarrow}(\lambda n)\right| P S_{\perp}\right\rangle
$$

Evidently, $h_{1}(x)$ counts transversely polarized quarks, in a transversely polarized nucleon.

As can be seen in equation (2.32), $h_{1}(x)$ is chiral-odd. Reference to figure 2.3 shows $h_{1}$ does not contribute to the forward scattering amplitude (in the massless limit), since the gauge interactions conserve chirality. Thus, $h_{1}$ is inaccessible in inclusive deepinelastic lepton scattering, and as a result, has not yet been measured. Indeed, until recently, it had hardly been noticed in the literature. In general, however, it contributes to hard processes at leading order in $1 / Q^{2}[4]$, and thus is on equal footing with the better known distributions, $f_{1}(x)$ and $g_{1}(x)$.

The moments of $h_{1}(x)$ can be related to matrix elements of local operators. The object of study in this thesis is the lowest moment, the so-called tensor charge:

$$
\delta q \equiv \int_{-\infty}^{\infty} d x h_{1}(x)=\frac{1}{P+\sqrt{2}}\left\langle P S_{\perp}\left|\psi_{+}^{\dagger} \gamma_{\perp} \gamma^{5} \psi_{+}\right| P S_{\perp}\right\rangle
$$

The name 'tensor charge' derives from the relation between $\delta q$ and the following matrix element:

$$
\left\langle P S\left|\bar{\psi} \sigma_{\mu \nu} i \gamma^{5} \psi\right| P S\right\rangle=\frac{2}{M_{T}}\left(P_{\mu} S_{\nu}-P_{\nu} S_{\mu}\right) \delta q
$$

Unlike the charges associated with the ordinary vector and axial-vector currents, $\delta q$ is not conserved. The tensor operator appearing in the matrix element in equation (2.37) has nonzero anomalous dimension, and as a result, $\delta q$ depends on the renormalization scale at which the operator is renormalized. This presents problems when we come to estimate $\delta q$ 
in models, since we generally do not know the renormalization scale that should apply to the model estimate. In the nonrelativistic quark model, for example, the tensor charges are equal to the corresponding axial charges (see section 4.). Jaffe and Ji [1] have shown that in the bag model, the tensor charges are somewhat larger in absolute magnitude than their axial charge counterparts. Unfortunately, these comparisons involve implicit, unknown renormalization scales (of order $1 \mathrm{GeV}$, in the case of the bag model). The scale information obtained from these comparisons is therefore of dubious value. The predictions for relative sizes, however, are likely to be more reliable, particularly in the case of the tensor charges; since they all share the same anomalous dimension, the ratio of, say, $\delta d$ to $\delta u$ is independent of the renormalization scale. In this thesis, I will use the Skyrme model to obtain estimates for $\delta d / \delta u$ and $\delta s / \delta u$. 


\section{THE SKYRME MODEL}

The Skyrme model is a semi-classical model that treats baryons as solitons in a non-linear, effective field theory of mesons. It has met with some success in describing the longdistance properties of the low-lying baryons [11]. 'Long-distance' properties are those which can be studied with low energy probes, which, by virtue of their long wavelengths, do not resolve the detailed structure of of the target. The soliton picture is not likely to give reasonable predictions for short-distance properties; after all, baryons are made of quarks and gluons, not mesons. A question immediately arises as to whether the tensor charges are 'long-distance properties' or 'short-distance properties'. In this thesis, we will use the Skyrme model only to calculate the ratios $\delta d / \delta u$ and $\delta s / \delta u$. As mentioned earlier, the tensor charges are all characterized by the same anomalous dimension, so these ratios are independent of the renormalization scale. The energy scale of the probe is therefore irrelevant.

Before reviewing the Skyrme model, a few comments are in order. In its original form, the Skyrme model was based on a specific lagrangian, so the resulting solitons had a specific spatial structure. In fact there are many equally acceptable lagrangians from which to begin, each of which leads to solitons with distinct spatial structure. We will be interested only in the $\mathrm{SU}(3)$ group properties of the tensor charges, however, and for this purpose, the various soliton models are equivalent to one another. For simplicity, the name 'Skyrme model' will be used to refer to the entire family of chiral soliton models. The second comment relates to the sophisticated (and elegant) mathematics used to study the Skyrme model. I will not attempt to present a rigorous exposition of these methods. Instead, in the interest of clarity, I will use a simplified approach, 
switching back and forth between the SU(2) and SU(3) versions of the model, and in some cases simply quoting results. A more rigorous exposition can be found in the article by Guadagnini [12].

The Skyrme model starts with a non-linear, effective lagrangian describing the interactions of the eight, light, pseudoscalar mesons. (These are the Goldstone bosons associated with the spontaneous breakdown of chiral $\left.\mathrm{SU}(3)_{\text {right }} \times \mathrm{SU}(3)_{\text {left }}.\right)$ The effective lagrangian must of course respect the symmetries of QCD. One such lagrangian takes the form

$$
\mathcal{L}=\frac{F_{\pi}^{2}}{16} \operatorname{Tr}\left(\partial_{\mu} U \partial^{\mu} U^{\dagger}\right)
$$

where $U=\exp \left(2 i \lambda^{a} \phi^{a} / F_{\pi}\right)$. The $\phi^{a}$ 's are meson fields, and the $\lambda^{a}$ 's are Gell-Mann matrices. The field equations derived from the lagrangian in equation (3.1) have a very interesting property: The space of classical solutions has non-trivial topology. To be specific, the classical solutions fall into disjoint subspaces. This raises the possibility of stable, non-trivial field configurations, i.e., solitons.

To elucidate the topology of the system, I will consider the simpler, $\mathrm{SU}(2)$ version of the model. The topology is identical [13], and is more readily visualized. Let $U(\mathbf{x})$ be a time-independent solution to the classical equations of motion. $U(\mathbf{x})$ can be regarded as a mapping from $\mathbf{R}^{3}$ to the group space of $\mathrm{SU}(2)$. If our solution is to have finite energy, $U(\mathbf{x})$ must approach some constant value at infinity. In other words, every point at infinity must be mapped into the same value of $\mathrm{SU}(2)$. It is sensible, therefore, to identify every point at infinity as 'the same point'. With this constraint, $\mathbf{R}^{\mathbf{3}}$ is isomorphic to $\mathbf{S}^{3}$, the three-sphere. (This isomorphism can be established by means of the threedimensional equivalent of a stereographic projection, whereby $\mathbf{R}^{2}$ is mapped onto $\mathbf{S}^{2}$.) 
The group space of $\mathrm{SU}(2)$ is also isomorphic to $\mathrm{S}^{3}$. (If $U$ is an $\mathrm{SU}(2)$ matrix, there are real numbers $a_{0}, a_{1}, a_{2}$, and $a_{3}$ such that $a_{0}^{2}+a_{1}^{2}+a_{2}^{2}+a_{3}^{2}=1$, and $U=a_{0}+i a_{i} \tau_{i}$.) Thus, we are interested in the space of mappings from $\mathbf{S}^{3}$ to $\mathbf{S}^{3}$. More precisely, we would like to group these mappings into homotopy classes.

The simplest mapping from $\mathbf{S}^{3}$ to $\mathbf{S}^{3}$ is the trivial map, which maps all of $\mathbf{S}^{3}$ into a single point. A more interesting map is the 'identity' map, which maps every point of $\mathbf{S}^{3}$ into itself. These two maps are topologically distinct; they fall into different homotopy classes, distinguished by an integer 'covering number'. The covering number associated with the trivial map is zero, while the covering number associated with the identity map is unity. (It may help to consider the analogous case of mappings from $\mathbf{S}^{2}$ to $\mathbf{S}^{2}$.) Solutions with unit covering number are topologically stable, in the sense that they cannot 'decay' into the trivial solution. We might therefore suspect that these solutions are the solitons we are seeking. At this point, however, we run into a problem. Although the simple lagrangian of equation (3.1) provides a mechanism for topological stability, it fails to provide a mechanism for mechanical stability. As a result, the only time-independent solutions to the equations of motion are trivial solutions.

This claim is easy to establish. For a time-independent solution, the classical action is given by

$$
S=-\frac{F_{\pi}^{2}}{4 \pi} \int d^{4} x \operatorname{Tr}\left(\partial_{i} U \partial_{i} U^{\dagger}\right)=-E
$$

This must be invariant under small variations of the field variables. In particular, consider scale transformations of the form

$$
U(\mathbf{x}) \rightarrow \tilde{U}(\mathbf{x})=U(\lambda \mathbf{x}) .
$$


Under this transformation, $S \rightarrow \widetilde{S}=-E / \lambda$. According to Hamilton's principle, $\widetilde{S}$ must be stationary under small variations in $\lambda$, when $\lambda=1$. Differentiating with respect to $\lambda$ and setting $\lambda$ equal to one, we obtain $E=0$, which of course implies that $U$ is constant. Skyrme was aware of this problem, and remedied it by adding a new term to the original lagrangian. The so-called 'Skyrme term' takes the form

$$
\mathcal{L}_{\text {Skyrme }}=\frac{1}{32 e^{2}} \operatorname{Tr}\left[\left(\partial_{\mu} U\right) U^{\dagger},\left(\partial_{\nu} U\right) U^{\dagger}\right]^{2} .
$$

It is straightforward to check explicitly that the Skyrme term does indeed stabilize the soliton. We can get a rough understanding of this by repeating our earlier analysis involving scale transformations. If we define $S=S_{\text {kinetic }}+S_{S k y r m e}$, where $S_{k i n e t i c}$ is the contribution to the action from the two-derivative, 'kinetic' term, then under the scale transformation above, $S \rightarrow-E_{k i n e t i c} / \lambda-\lambda E_{S k y r m e}$. This leads to the stability condition $E_{\text {kinetic }}=E_{S k y r m e}$, which is clearly consistent with a finite energy soliton solution.

The Skyrme term is clearly ad hoc, and is in fact, representative of a serious weakness of the model. Let me pose the following question: Having added a four-derivative term to the lagrangian, how do we know there are no terms with six derivatives? In the interest of continuity, I will sidestep this issue for the moment, but will return to it shortly.

It is easy to construct a time-independent, finite energy solution to the new system of equations. For the $\mathrm{SU}(2)$ version of the model, we begin with the so-called 'hedgehog' ansatz:

$$
U_{0}(\mathbf{x})=\exp [i F(r) \tau \cdot \hat{\mathbf{r}}]=\cos (F(r))+i \tau \cdot \hat{\mathbf{r}} \sin (F(r))
$$

If $U_{0}(\mathbf{x})$ is to be well-defined at $x=0$, we must take $F(0)=n \pi$, where $n$ is an integer. It will turn out that $n$ is the covering number, and since we are interested in single 
baryon states, we will take $n=1$. If $U_{0}(\mathbf{x})$ is to have finite energy, $F(r)$ must vanish as $r$ approaches infinity. Thus, $\mathbf{x}=0$ is mapped into -1 , and the point at infinity is mapped into +1 , where 1 is the identity element of $\mathrm{SU}(2)$. If we write $U_{0}=a_{0}+i a_{i} \tau_{i}$, with $a_{0}^{2}+a_{1}^{2}+a_{2}^{2}+a_{3}^{2}=1$, we see that $\mathbf{x}=0$ is mapped into the 'south pole' of $\mathbf{S}^{3}$ (i.e., $a_{0}=-1, a_{1}=a_{2}=a_{3}=0$ ), while the point at infinity is mapped into the 'north pole'. This is the three-dimensional analog of a stereographic projection, whereby $\mathbf{R}^{2}$ is mapped onto $\mathbf{S}^{2}$. The covering number of this map is unity. The radial function $F(r)$ can be determined by minimizing the soliton energy, subject to the boundary conditions given above. I will not carry out this calculation, since it must be done numerically, and is not particularly enlightening. The interested reader should refer to reference [11].

The non-trivial topology of the Skyrme model gives rise to a topological conservation principle: Solutions of a given covering number cannot 'decay' into solutions of a different covering number. The covering number can thus be thought of as a conserved 'topological charge'. In fact, there is a conserved current associated with this charge:

$$
J_{\mu}=\frac{1}{24 \pi^{2}} \epsilon_{\mu \nu \alpha \beta} \operatorname{Tr}\left[U^{\dagger}\left(\partial^{\nu} U\right) U^{\dagger}\left(\partial^{\alpha} U\right) U^{\dagger}\left(\partial^{\beta} U\right)\right]
$$

Notice that $J_{\mu}$ is trivially conserved, irrespective of the dynamics of $U$. It can be shown that for any solution of the classical field equations, the topological charge, $N \equiv \int d^{3} x J_{0}(\mathbf{x})$, is an integer [13]. (For the hedgehog solution above, with $F(0)=n \pi$ and $F(\infty)=0$, we obtain $N=n$.)

At this point, it is tempting to regard the unit covering number soliton as a baryon. Is there any evidence to support this idea? In particular, can we show that 'covering number' equals 'baryon number'? I will describe a heuristic method, due to Goldstone and Wilczek, which can be used to establish this equality. 
Traditionally, we derive conserved currents by means of Noether's theorem. We start with an invariance of the lagrangian, make an appropriate infinitesimal transformation of the field variables, and demand that the lagrangian remain unchanged. Unfortunately, the symmetry transformation that is of interest here is $U \rightarrow e^{i \theta} U e^{-i \theta}$, where $\theta$ is a real number. This is the $U(1)$ symmetry associated with baryon number conservation, and the lagrangian is trivially invariant under this transformation. How do we proceed?

Goldstone and Wilczek [14] found a heuristic way to derive the desired baryon number current. The idea is to couple fermion fields to the meson fields, then compute the expectation value of the fermion number (i.e., baryon number) current in the presence of the classical soliton field. Let $\psi$ be a triplet of massless fermion fields. We will use the following lagrangian:

$$
\mathcal{L}=\bar{\psi} i \gamma^{\mu} \partial_{\mu} \psi+m\left(\bar{\psi}_{L} U \psi_{R}+\bar{\psi}_{R} U^{\dagger} \psi_{L}\right)
$$

There is no kinetic term for the meson fields because we are treating them as classical, external fields. The baryon number current is easy to identify: $J_{\mu}=\bar{\psi} \gamma_{\mu} \psi$. The next step is to compute the 'vacuum expectation value' of $J_{\mu}$ in the presence of the classical meson fields. The result will be some function of the meson field variables, and will be taken as the desired baryon number current. The calculation is simple, and can be found in Goldstone and Wilczek's paper. The result is identical to the conserved topological current given above:

$$
\begin{aligned}
J_{\mu} & =\left\langle 0\left|\bar{\psi} \gamma_{\mu} \psi\right| 0\right\rangle_{S} \\
& =\frac{1}{24 \pi^{2}} \epsilon_{\mu \nu \alpha \beta} \operatorname{Tr}\left[U^{\dagger}\left(\partial^{\nu} U\right) U^{\dagger}\left(\partial^{\alpha} U\right) U^{\dagger}\left(\partial^{\beta} U\right)\right] .
\end{aligned}
$$

It begins to seem reasonable to regard the unit covering number soliton as a baryon. 
It is now time to deal with the issue of higher-derivative terms. Thus far, we have arbitrarily excluded terms with more than four derivatives. A bit of thought shows that this is not a reasonable approximation. The chiral lagrangian with which we started is non-renormalizable, so we must include terms with arbitrarily large numbers of derivatives, to serve as counterterms. Normally, these terms do not pose a problem, because we use the chiral lagrangian only to study the interactions of low energy mesons. Every derivative comes with an inverse factor of some large mass parameter (so that every term in the lagrangian will have the same mass-dimension). This factor can be shown to be approximately equal to $4 \pi F_{\pi}$, which is of order $1 \mathrm{GeV}$ [15]. Thus, if we work with low energy mesons, higher-derivative terms are suppressed, since $\partial_{\mu} / 4 \pi F_{\pi}$ gets traded for $p_{\mu} / 4 \pi F_{\pi}$. In the case of the hedgehog, however, this nice derivative expansion breaks down, because the classical meson fields are rapidly varying near the origin. It is not true that $\partial_{\mu} / 4 \pi F_{\pi}$ is a small number, so we have no basis upon which to ignore higherderivative terms in the Skyrme model. (Recall that the stability condition we derived earlier requires $E_{S k y r m e}=E_{k i n e t i c}$. If the so-called derivative expansion were valid for the Skyrme model, we would expect the four-derivative Skyrme term to make a small contribution to the soliton energy, relative to the two-derivative 'kinetic' term.)

We seem to faced with a problem. If we are forced to include terms with an arbitrarily large number of derivatives, will we not lose all predictive power? And how do we know the soliton is still energetically stable? I will show how to handle the first problem momentarily, but for now, let me touch on the second problem.

Witten, t'Hooft, and others have studied QCD in the limit as the number of colors, $N_{c}$, becomes large. (For a review, see reference [16].) Their studies suggest that in 
the large- $N_{c}$ limit, QCD becomes a theory of weakly interacting mesons. Witten [16] proposed a way to incorporate baryons into the picture, and showed that his baryons had the properties of solitons. Thus, although the original Skyrme model may not give the correct predictions for baryon matrix elements, the evidence suggests that in the large$N_{c}$ limit, there is some chiral soliton model which does. In fact, we will see that when soliton models are quantized semi-classically, through the use of collective coordinates, they are all equivalent, in the following sense: The baryon matrix elements of any given QCD operator differ from one chiral soliton model to the next only by a single (unknown) scale factor for each operator. (In view of this equivalence, all chiral soliton models will henceforth be referred to collectively as the Skyrme model.)

In order to compute baryon matrix elements in the Skyrme model, we will need to quantize the soliton. But what do we quantize? There is no obvious set of variables to which we can apply the standard canonical quantization procedure. To get around this problem, we begin by observing that the soliton field configuration is a classical field configuration. We expect the quantum soliton field to exhibit fluctuations about the classical configuration. The variables describing these fluctuations are the objects that we must quantize.

We are interested in the low-lying states of the soliton, so we should concentrate on fluctuations that involve the least amount of energy. If we imagine decomposing fluctuations in the soliton field in terms of normal modes of oscillation, then we should concentrate on the normal modes with the lowest frequencies. In fact, there is a class of 'oscillations' which involve no restoring forces, and hence are candidates for the lowest energy quantum fluctuations. Notice that if $U_{0}$ is a solution to the classical field equations, then $A U_{0} A^{\dagger}$ is also a solution, for any constant $\mathrm{SU}(3)$ matrix $A$. This symmetry 
gives rise to a set of so-called 'zero modes', which in this case are essentially rigid rotations of the soliton field configuration. We will take $A$ as a collective variable, which we will then quantize. In so doing, we will restore the $\mathrm{SU}(3)_{\text {flavor }} \times \mathrm{SU}(2)_{\text {spin }}$ symmetry which was broken by our specific choice of a classical solution. It is important to remember that this 'semi-classical' quantization procedure ignores all other excitations of the soliton field, and therefore assumes implicitly that the rotational kinetic energy is small.

To construct the lagrangian describing the dynamics of the collective variable $A$, we begin by defining the time-dependent, rotating soliton field, $U(\mathbf{x}, t)=A(t) U_{0}(\mathbf{x}) A^{\dagger}(t)$, where $U_{0}(\mathbf{x})$ is the hedgehog solution. We then insert $U(\mathbf{x}, t)$ into the lagrangian, and integrate out the spatial dependence. For the $\mathrm{SU}(2)$ version of the model, we obtain

$$
L(A(t), \dot{A}(t))=-M+\lambda\left(\dot{a}_{0}^{2}+\dot{a}_{1}^{2}+\dot{a}_{2}^{2}+a_{3}^{2}\right),
$$

where we have used $A(t)=a_{0}(t)+i a_{i}(t) \tau_{i}$. The term $M$ comes from the time-independent piece of the meson lagrangian, $\mathcal{L}\left(U, U^{\dagger}\right)$, and represents the classical soliton energy. $M$ and $\lambda$ depend on the specific form of the meson lagrangian, but the generic form of $L(A, \dot{A})$ given above is the only form which is consistent with $\mathrm{SU}(2)$ invariance and which contains no more than two time derivatives. (Note: $a_{0} \ddot{a}_{0}+a_{i} \ddot{a}_{i}=-\dot{a}_{0}^{2}-\dot{a}_{i} \dot{a}_{i}$. We are assuming that the soliton rotates slowly, so we can neglect terms involving three or more time-derivatives.)

At this point, the quantization procedure is straightforward. We compute $\partial L / \partial \dot{a}_{i}$, and identify this with $p_{i}$, the momentum conjugate to $a_{i}$. We then construct the hamiltonian as $H=\left(p_{0} \dot{a}_{0}+p_{i} \dot{a}_{i}\right)-L$, and quantize in the usual manner, using canonical commutators. (With a few modifications, essentially the same procedure works for the SU(3) 
version of the model.) As mentioned above, however, this procedure is unnecessary. We can construct the collective coordinate energy eigenfunctions directly, without reference to a specific hamiltonian. The key lies in the constraint equation: $a_{0}^{2}+a_{1}^{2}+a_{2}^{2}+a_{3}^{2}=1$. Imagine a classical particle constrained to move on the surface of a unit sphere in three dimensions. The particle coordinates satisfy the constraint $x_{1}^{2}+x_{2}^{2}+x_{3}^{2}=$ 1. As long as the hamiltonian does not break the spherical symmetry of the system, the energy eigenstates can be constructed without reference to the hamiltonian. The angular dependence of the eigenstates is determined by the requirement that they carry irreducible representations of the rotation group. Since there is no radial degree of freedom, the eigenstates are completely determined. Precisely the same result holds in the Skyrme model. The energy eigenstates are completely determined by the assumed SU(3) invariance of the hamiltonian and the constraint that the collective variable $A$ lie on the $\mathrm{SU}(3)$ manifold.

As a warmup for the calculation of baryon matrix elements in the Skyrme model, I will consider the closely analogous example of a particle on the surface of a sphere. In the $\mathrm{SU}(2)$ Skyrme model, the hedgehog can be thought of as having coordinates $a_{0}=1, a_{1}=a_{2}=a_{3}=0($ i.e., $A=1$ ). The analogous state for a particle on a sphere is $|\hat{z}\rangle$, which represents a particle located at the north pole. This state does not have welldefined angular quantum numbers, but we can construct such states using projection operators. Consider the following operator:

$$
P_{m_{1} m_{2}}^{J}=(2 J+1) \int_{S U(2)} d g D_{m_{1} m_{2}}^{* J}(g) U(g)
$$


where $D_{m_{1} m_{2}}^{J}(g)$ is the representation matrix associated with group element $g$, and $U(g)$ is the corresponding unitary operator on the hilbert space. $P_{m_{1} m_{2}}^{J}$ is a projection operator. If we write $|\hat{z}\rangle$ as a superposition, $\sum_{j m} a_{j m}|j m\rangle$, of angular momentum eigenstates, then the orthogonality of the group representation matrices can be used to show that

$$
P_{m_{1} m_{2}}^{J}|\hat{z}\rangle=a_{J m_{2}}\left|J m_{1}\right\rangle
$$

We would like to determine the spectrum of states in the hilbert space. In other words, what states can we create from $|\hat{z}\rangle$ ? From equation (3.11), we see that we can create states of any $\mathrm{SU}(2)$ representation $J$, provided $J$ contains a state $|J m\rangle$ satisfying

$$
a_{J m}=\langle J m \mid \hat{z}\rangle \neq 0
$$

To determine the conditions under which $a_{J m} \neq 0$, we observe that $|\hat{z}\rangle$ is an eigenstate of $J_{z}$, with eigenvalue zero. Thus, $\langle J m \mid \hat{z}\rangle$ must vanish unless $m=0$. It is easy to show that this is also a sufficient condition that $a_{J m}$ be non-vanishing. Since every representation with integer spin contains a state with $m=0$, the hilbert space carries all representations with integer spin.

Now consider a more interesting case. Suppose we give the particle a charge $e$, and place a magnetic monopole at the origin. The system is still spherically symmetric, so the energy eigenstates still form representations of $\mathrm{SU}(2)$. We will find, however, that there are now restrictions on the allowed representations. In the presence of the monopole, the generators of rotations become

$$
\mathbf{J}=\mathbf{x} \times(\mathbf{p}-e \mathbf{A})-e g \mathbf{x}
$$


where $g$ is the magnetic charge of the monopole. It is well known that the product eg must be an integer or a half-integer [29]. (This is the Dirac quantization condition.) The state $|\hat{z}\rangle$ is still an eigenstate of $J_{z}$, but it now has eigenvalue -eg. Thus, $\langle J m \mid \hat{z}\rangle$ must vanish unless $m=-e g$. Only representations with $J \geq|e g|$ can contain states with $m=-e g$. Thus, we recover the well-known fact that in the presence of a monopole, only states with angular momentum $J \geq|e g|$ are allowed.

Finally, we want to evaluate matrix elements of the form $\left\langle j^{\prime} m^{\prime}\left|O_{J M}\right| j m\right\rangle$, where $O_{J M}$ is a tensor operator belonging to the spin- $J$ representation of $\mathrm{SO}(3)$. For reasons to be discussed shortly, we are particularly interested in operators that do not involve the canonical momenta. In other words, we will assume that $O_{J M}$ depends only on the coordinates $x, y$, and $z$. Since the spherical harmonics are a complete set of orthonormal functions on the unit sphere, $O_{J M}$ must be a constant multiple, $k$, of $Y_{J M}$. The matrix element is now easily evaluated:

$$
\begin{aligned}
\left\langle j^{\prime} m^{\prime}\left|O_{J M}\right| j m\right\rangle & =k\left\langle j^{\prime} m^{\prime}\left|Y_{J M}\right| j m\right\rangle \\
& =k \sqrt{\frac{(2 J+1)(2 j+1)}{4 \pi\left(2 j^{\prime}+1\right)}}\left\langle J j ; j^{\prime} m^{\prime} \mid J M ; j m\right\rangle\left\langle J j ; j^{\prime} 0 \mid J 0, j 0\right\rangle .
\end{aligned}
$$

The first Clebsch-Gordan coefficient was expected, on the basis of the Wigner-Eckart theorem. Note, however, that the constant $k$ depends only on operator $O_{J}$, and is independent of the representation labels $j$ and $j^{\prime}$. This will be particularly important in the SU(3) Skyrme model, because a given SU(3) representation may occur more than once in a direct product representation.

Now for the Skyrme model. This will be an almost verbatim repetition of the preceding analysis. Before I begin, however, it will be necessary to introduce the socalled Wess-Zumino effective action. 
The Wess-Zumino term [17] is an essential ingredient in any low energy effective theory of mesons. It cannot be written as an integral over four-dimensional spacetime, but must instead be written as an integral over a five-dimensional surface, $Q$, whose boundary is spacetime:

$$
\Gamma_{W Z}=-\frac{i}{240 \pi^{2}} \int_{Q} d^{5} x \epsilon^{\mu \nu \alpha \beta \gamma} \operatorname{Tr}\left(U^{\dagger} \partial_{\mu} U \ldots U^{\dagger} \partial_{\gamma} U\right)
$$

$\Gamma_{W Z}$ appears in the effective action along with a multiplicative constant, $N$, which can be shown to be quantized in integer units. This term was originally introduced to account for QCD anomalies, of the sort that give rise to $\pi^{0} \rightarrow 2 \gamma$. (By normalizing this decay amplitude, it can be shown that $N=N_{c}$.) It is important because it is the lowest dimensional term that allows such decays, while preserving the chiral symmetry of the effective theory. Witten has written a very insightful paper discussing the significance of the Wess-Zumino term [18]. For our purposes, the most important result of his analysis is his observation of the close analogy between the Wess-Zumino term and the term that must be added to the particle-on-a-sphere example in order to account for a monopole at the center. The requirement that the coefficient $N$ be an integer is directly related to the requirement that $2 e g$ be an integer. And just as the monopole places restrictions on the allowed representations of SU(2), the Wess-Zumino term places restrictions on the representations of $\mathrm{SU}(3) \times \mathrm{SU}(2)$ that can be realized in the Skyrme model.

We begin with the hedgehog solution, which is the Skyrme model analog of $|\hat{z}\rangle$. To emphasize the analogy, I will denote this state by $|Z\rangle$. As in the previous example, states with well-defined $\mathrm{SU}(3) \times \mathrm{SU}(2)$ quantum numbers can be created by means of 
projection operators. Let $R$ be an SU(3) representation label, and let $a$ denote a complete set of SU(3) quantum numbers. Then we can write $|Z\rangle$ as a superposition:

$$
|Z\rangle=\sum a_{R a ; J m}|R a ; J m\rangle .
$$

The hilbert space will contain representation $R \times J$, provided that this representation contains a state $|R a ; J m\rangle$ such that

$$
a_{R a ; J m}=\langle R a ; J m \mid Z\rangle \neq 0 .
$$

Again, as in the previous example, we can determine the conditions under which this is non-vanishing by considering the set of generators which leave $|Z\rangle$ invariant. The generators having this property can be shown to be $\mathbf{I}+\mathbf{J}$ and $Y[19]$ :

$$
\begin{aligned}
& (\mathbf{I}+\mathbf{J})|Z\rangle=0 \\
& Y|Z\rangle=\frac{N_{c}}{3}|Z\rangle
\end{aligned}
$$

( $\mathbf{I}$ and $\mathbf{J}$ are the isospin and spin generators, respectively, and $Y$ is the hypercharge.) The first equation might have been guessed by considering the special form of the hedgehog solution. The second equation shows the effects of the Wess-Zumino term. If not for this term, the right-hand side of equation (3.19) would vanish. Equation (3.19) requires that $|R a ; J m\rangle$ have hypercharge $Y=N_{c} / 3$. Equation (3.18), however, must be manipulated to obtain a condition on $|R a ; J m\rangle$, since this state is not an eigenstate of $\mathrm{I}+\mathrm{J}$. The following two equations are trivial consequences of equation (3.18):

$$
\begin{aligned}
& \langle R a ; J m|\mathbf{I} \cdot(\mathbf{I}+\mathbf{J})| Z\rangle=\left\langle R a ; J m\left|\mathbf{I}^{2}+\mathbf{I} \cdot \mathbf{J}\right| Z\right\rangle=0 \\
& \langle R a ; J m|\mathbf{J} \cdot(\mathbf{I}+\mathbf{J})| Z\rangle=\left\langle R a ; J m\left|\mathbf{J}^{2}+\mathbf{J} \cdot \mathbf{I}\right| Z\right\rangle=0
\end{aligned}
$$


Since $[\mathbf{I}, \mathbf{J}]=0$, we conclude that the spin and isospin representation labels, $I$ and $J$, must be equal. Summarizing, then, every representation in the hilbert space must contain a state with $I=J$, and $Y=N_{c} / 3$.

We now face the task of computing matrix elements in the Skyrme model. This will be considerably easier if we work in the large- $N_{c}$ limit. In fact, consistency demands that we take $N_{c}$ to be large. In quantizing the zero-modes of the soliton, we assumed that all other quantum fluctuations were negligible by comparison. Since the spin of the soliton is quantized in units of $1 / 2$, this requires the moment of inertia of the soliton to be large, so that the rotational kinetic energy will be small. On general grounds, the soliton moment of inertia can be shown to be proportional to $N_{c}$, in the large- $N_{c}$ limit [16], so our semi-classical quantization procedure assumes implicitly that we are working in the large- $N_{c}$ limit.

In order to compute matrix elements in the Skyrme model, one must first identify an appropriate Skyrme model operator to serve as an analog for the QCD operator in question. This operator must of course be constructed in terms of meson fields. In general, there will be an infinite family of meson operators having the right transformation properties to serve as analog operators. Aside from rare exceptions, there is no known method of choosing the correct combination of these operators. In the large- $N_{c}$ limit, however, it can be argued that the contributions of meson operators involving time-derivatives can be neglected. This leads to a greát simplification. The basic idea is that time-derivatives bring in factors of the soliton rotation frequency, which tends to zero in the large- $N_{c}$ limit. To be more quantitative, we can compare the contribution of an operator involving a time-derivative with that of a similar operator which contains 
a space-derivative in place of the time-derivative. Meson operators are reduced to collective coordinate operators by integrating out the spatial dependence. This brings in a factor of the order of the inverse proton radius for each space-derivative. Thus, we should compare the soliton rotation frequency, $\omega$, to the inverse proton radius, $1 / R$ :

$$
\frac{\omega}{1 / R} \sim \frac{J / I}{1 / R} \sim \frac{J}{M R}
$$

The angular momentum of the spinning soliton is denoted by $J$, while $M$ and $I$ denote the mass and moment of inertia, respectively. Baryon masses grow as $N_{c}$, while their radii remain constant in the large- $N_{\mathrm{c}}$ limit [16]. Thus, we conclude that the contributions of operators involving time-derivatives are suppressed by $1 / N_{c}$.

In view of the previous analysis, we can restrict our attention to collective coordinate operators that do not involve factors of the canonical momenta. At this point, the calculation of matrix elements becomes a straightforward exercise in group theory. I will sketch the calculation briefly; the details can be found in the article by Manohar [19]. We will work in the $\mathrm{SU}(3)$ equivalent of the position-space basis of ordinary quantum mechanics. With each value of the collective variable, $A$, we associate a state $|A\rangle$ belonging to the hilbert space. These states form a complete, orthonormal basis. The 'wavefunction' of state $|\Psi\rangle$ is given by

$$
\Psi(A)=\langle A \mid \Psi\rangle
$$

Since the SU(3) group representation matrices are a complete set of functions on the $\mathrm{SU}(3)$ manifold, we can write $\Psi(A)$ in terms of representation matrices. The $\mathrm{SU}(3) \times$ 
$\mathrm{SU}(2)$ transformation properties of $A$ can then be used to show that the wavefunction for the state $|R a ; J m\rangle$ is given by

$$
\langle A \mid R a ; J m\rangle=\sqrt{\operatorname{dim} R} D_{a, b}^{R}(A) .
$$

The label $b$ denotes an SU(3) state with quantum numbers $I=J, I_{3}=-m$, and $Y=$ $N_{c} / 3$. Since the operators of interest depend only on the coordinates, they can also be written in terms of representation matrices. Matrix elements can then be computed, up to a single unknown multiplicative factor, by using the orthogonality of the representation matrices and the Clebsch-Gordan decomposition of direct product representations:

$$
\begin{aligned}
& \left\langle R_{2} a_{2} ; J_{2} m_{2}\left|O_{R a ; J m}\right| R_{1} a_{1} ; J_{1} m_{1}\right\rangle=k \int d A D_{a_{2}, b_{2}}^{* R_{2}}(A) D_{a, b}^{R}(A) D_{a_{1}, b_{1}}^{R_{1}}(A) \\
& =k(-1)^{J_{1}+J_{2}-m_{1}-m_{2}}\left(\frac{\operatorname{dim} R_{1}}{\operatorname{dim} R_{2}}\right)^{1 / 2} \sum_{n}\left(\begin{array}{cc|c}
R_{1} & R & R_{2_{n}} \\
a_{1} & a & a_{2}
\end{array}\right)\left(\begin{array}{cc|c}
R_{1} & R & R_{2_{n}} \\
b_{1} & b & b_{2}
\end{array}\right) .
\end{aligned}
$$

The label $b$ denotes an $\mathrm{SU}(3)$ state with $I=J, I_{3}=-m$, and $Y=0 ; b_{1}$ denotes an SU(3) state with $I=J_{1}, I_{3}=-m_{1}$, and $Y=N_{c} / 3 ; b_{2}$ denotes an SU(3) state with $I=J_{2}, I_{3}=-m_{2}$, and $Y=N_{c} / 3$. The sum is over all occurrences of $R_{2}$ in the direct product $R_{1} \times R$. It is important to observe that the constant $k$ depends only on operator $O_{R J}$, and is completely independent of the states $\left|R_{1} a_{1} ; J_{1} m_{1}\right\rangle$ and $\left|R_{2} a_{2} ; J_{2} m_{2}\right\rangle$. 


\section{CALCULATION OF THE TENSOR CHARGES}

Before discussing the Skyrme model calculation, it will be useful to consider the tensor charges in the non-relativistic quark model (NQM). In the NQM, the tensor charges are equal to the axial charges. This is true for any value of $N_{c}$. The calculation described below is due to Karl and Paton [20]. It proceeds from the observation that the axial charges measure the spin carried by quarks.

In the rest frame of the proton, the tensor charge, $\delta q$, is given by

$$
2 S^{i} \delta q=\left\langle P S\left|\bar{q} \sigma^{i 0} i \gamma^{5} q\right| P S\right\rangle=\left\langle P S\left|\bar{q} \sigma_{i} q\right| P S\right\rangle
$$

The corresponding axial charge, $\Delta q$, is given by

$$
2 S^{i} \Delta q=\left\langle P S\left|\bar{q} \gamma^{i} \gamma^{5} q\right| P S\right\rangle=\left\langle P S\left|q^{\dagger} \sigma_{i} q\right| P S\right\rangle
$$

In the NQM, the small components of the quark spinors vanish, so we can replace $\bar{q} \sigma_{i} q$ with $q^{\dagger} \sigma_{i} q$ in equation (4.1). This leads to the immediate result that $\Delta q=\delta q$ in the NQM. The operators $S_{i}^{(q)} \equiv \frac{1}{2} q^{\dagger} \sigma_{i} q$ are the generators of rotations for the spin degrees of freedom, for quarks of type $q$. Since the quarks have no orbital angular momentum in the NQM version of a proton, we then obtain the following equations:

$$
\begin{aligned}
& S^{i} \Delta u=\left\langle P S\left|J_{i}^{(u)}\right| P S\right\rangle \\
& S^{i} \Delta d=\left\langle P S\left|J_{i}^{(d)}\right| P S\right\rangle,
\end{aligned}
$$

where $J_{i}^{(u)}$ is the generator of rotations for up quarks, and $J_{i}^{(d)}$ is the corresponding generator for down quarks. The NQM version of a proton contains no strange quarks, so $\Delta s=\delta s=0$. Thus, the up and down quarks account for all of the proton spin, and we have $\mathbf{J}=\mathbf{J}^{(u)}+\mathbf{J}^{(d)}$ 
To proceed, it will be necessary to discuss the symmetry properties of the proton wavefunction under interchange of quark pairs. (This will also be useful when we come to the Skyrme model.) A proton consisting of $N_{c} \equiv 2 n+1$ quarks must contain $n+1$ up quarks and $n$ down quarks if it is to have isospin $1 / 2$ and strangeness zero. In the NQM version of a proton, all quarks are in the same spatial state, which implies that the spatial wavefunction is symmetric under interchange of any pair of quarks. The color wavefunction, on the other hand, is assumed to be totally antisymmetric. Since the complete wavefunction must be totally antisymmetric, we conclude that the flavorspin wavefunction must be totally symmetric. Clearly, the flavor wavefunction must be symmetric under interchange of any pair of up quarks, so we conclude that the $n+1$ up quarks are in a totally symmetric spin state; in other words, the total spin quantum number of the up quarks must be $(n+1) / 2$. Similarly, the total spin quantum number of the down quarks must be $n / 2$.

We can now use the projection theorem to calculate $\Delta u$ and $\Delta d$ :

$$
\begin{aligned}
\Delta u & =\left\langle P \uparrow\left|J_{z}^{(u)}\right| P \uparrow\right\rangle \\
& =\left\langle P \uparrow\left|J_{z}\right| P \uparrow\right\rangle \frac{\left\langle P \uparrow\left|\mathbf{J} \cdot \mathbf{J}^{(u)}\right| P \uparrow\right\rangle}{\left\langle P \uparrow\left|\mathbf{J}^{2}\right| P \uparrow\right\rangle} \\
& =\frac{N_{c}+5}{12} \\
\Delta d & =\left\langle P \uparrow\left|J_{z}^{(d)}\right| P \uparrow\right\rangle \\
& =\left\langle P \uparrow\left|J_{z}-J_{z}^{(u)}\right| P \uparrow\right\rangle \\
& =\frac{1-N_{c}}{12}
\end{aligned}
$$

Assembling these results, we obtain

$$
\begin{aligned}
& g_{A}^{3}=2(\Delta u-\Delta d)=\frac{N_{c}+2}{3} \\
& g_{A}^{8}=\frac{g_{A}^{0}}{\sqrt{2}}=\frac{2}{\sqrt{3}}(\Delta u+\Delta d-2 \Delta s)=\frac{1}{\sqrt{3}} .
\end{aligned}
$$


The tensor charges are given by $g_{T}^{a}=g_{A}^{a}$. It must be remembered that the tensor charges are not conserved, so this calculation involves an implicit, unknown renormalization scale (i.e., the scale at which the tensor charge operators are renormalized). Since the (octet) axial charges are conserved, the equality of axial and tensor charges can hold only at a single value of the renormalization scale. The ratios $\delta d / \delta u$ and $\delta s / \delta u$, however, are independent of the renormalization scale. The NQM values for these ratios are are given by

$$
\begin{aligned}
& \frac{\delta d}{\delta u}=-\left(\frac{N_{c}-1}{N_{c}+5}\right) \\
& \frac{\delta s}{\delta u}=0 .
\end{aligned}
$$

The known correspondence [19] between the large- $N_{c}$ NQM and the large- $N_{c}$ Skyrme model suggests that we should expect similar results in the Skyrme model.

We will use equation (3.24) to study the tensor charges in the Skyrme model, but before doing so, a few comments are in order. Ideally, we would like to evaluate the proportionality constant $k$ appearing in the equation. As we just discussed, however, the tensor charges are renormalization scale-dependent. Any calculation of $k$ within the framework of the Skyrme model would therefore be of limited value, since presumably we would not know the renormalization scale that should apply to such a calculation. Aside from this problem, there is a second, related difficulty, which was discussed in connection with equation (3.24). Since the tensor charges are not associated with any symmetry of QCD, we lack a reliable means of constructing the correct Skyrme model analogs for the tensor charge operators. (If the tensor charges were associated with a symmetry of QCD, we could use Noether's theorem to construct the analog operators directly from the Skyrme model lagrangian.) Therefore, we are forced to use equation (3.24) as it is, without any direct estimate for the scale factor $k$. 
Consider the following matrix element:

$$
\left\langle P S\left|\bar{\Psi} \lambda^{a} \sigma_{\mu \nu} i \gamma^{5} \Psi\right| P S\right\rangle=\frac{1}{M}\left(S_{\mu} P_{\nu}-S_{\nu} P_{\mu}\right) g_{T}^{a}
$$

For a nucleon at rest, the tensor operator appearing in this equation yields non-vanishing matrix elements only for $(\mu, \nu)=(i, 0), i=1,2,3$. Denote these components of the operator by $O_{i}^{a}$, and denote the equivalent Skyrme model operators by $\widetilde{O}_{i}^{a}$. The $\widetilde{O}_{i}^{a}$ transform as a three-vector under spatial rotations, and as an octet under $\mathrm{SU}(3)$ transformations (or as an SU(3) singlet, in the case of $a=0$.) Let $|P \uparrow\rangle$ denote a proton state, with spin polarized up along the $z$-axis. Then equation (3.24) gives the following result for the octet charges:

$$
\begin{aligned}
g_{T}^{a} & =\frac{1}{M}\left\langle P \uparrow\left|\widetilde{O}_{3}^{a}\right| P \uparrow\right\rangle \\
& =k \sum_{n=1,2}\left(\begin{array}{cc|c}
\mathbf{R} & \mathbf{8} & \mathbf{R}_{n} \\
P & a & P
\end{array}\right)\left(\begin{array}{cc|c}
\mathbf{R} & \mathbf{8} & \mathbf{R}_{n} \\
P & b & P
\end{array}\right)
\end{aligned}
$$

(The proton mass has been absorbed into the proportionality constant, $k$.) The label $b$ denotes an $\mathrm{SU}(3)$ state with $Y=0, I=1$, and $I_{3}=0$. The label $\mathbf{R}$ denotes the $\mathrm{SU}(3)$ representation containing the proton. (We will discuss the proper choice for $\mathbf{R}$ momentarily.) The direct product $\mathbf{R} \times \mathbf{8}$ is readily seen to contain two copies of the representation $\mathbf{R}$, which are labeled with the subscript $n$. The corresponding formula for the singlet charge is given by

$$
\begin{aligned}
g_{T}^{\mathbf{0}} & =\frac{1}{M}\left\langle P \uparrow\left|\widetilde{O}_{3}^{0}\right| P \uparrow\right\rangle \\
& =k\left(\begin{array}{ll|l}
\mathbf{R} & \mathbf{1} & \mathbf{R} \\
P & 0 & P
\end{array}\right)\left(\begin{array}{cc|c}
\mathbf{R} & \mathbf{1} & \mathbf{R} \\
P & b & P
\end{array}\right)
\end{aligned}
$$

As in the octet case, the label $b$ refers to a state with $Y=0, I=1$, and $I_{3}=0$. Since the singlet representation contains no such state, the right-hand side of equation 
(4.9) vanishes. It is important to remember that, although corrections to this result are 'suppressed' in the large- $N_{c}$ limit, this does not necessarily imply that $g_{T}^{0}$ vanishes as $N_{c} \rightarrow \infty$.

We now discuss the choice of representation for the proton. We will appeal to quark models to justify our decision. Assume that the proton is constructed from $N_{c}=2 n+1$ quarks. In most quark models, the states of lowest energy are obtained by placing all quarks in the same spatial state. As discussed earlier, this implies that the flavor-spin wavefunction is totally symmetric. Now consider the spin state. This can be thought of as belonging to the direct product of $N_{c}$ fundamental representations of $\mathrm{SU}(2)$. The various irreducible representations belonging to this direct product can be identified by means of Young tableau (see figure 4.1). As indicated in the figure, there is exactly one spin- $\frac{1}{2}$ representation belonging to this direct product.

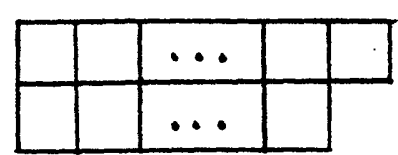

$$
J=\frac{1}{2}
$$

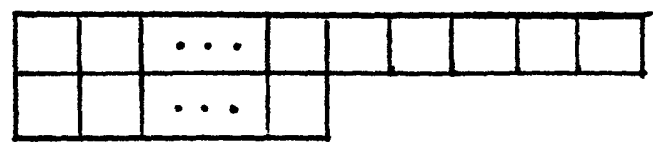

$$
J=\frac{5}{2}
$$

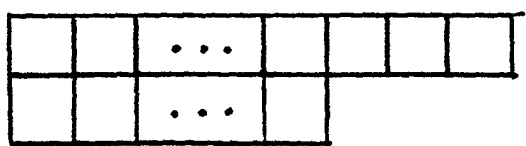

$J=\frac{3}{2}$

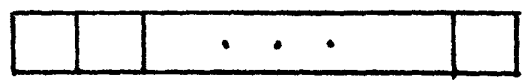

$$
J=\frac{N_{c}}{2}
$$

Fig. 4.1: Young Tableau for direct product of $N_{c}=2 n+1$ fundamental representations of SU(2). Each tableau contains $N_{c}$ boxes.

The Young tableau corresponding to a given representation specifies the symmetry properties of the states belonging to the representation [21]. For example, a pair of verti- 
cally stacked boxes represents a pair of spins in an antisymmetric, spin-singlet state. If a pair of quarks are in an antisymmetric spin state, they must also be in an antisymmetric flavor state if the flavor-spin wavefunction is to be symmetric under interchange of any pair of quarks. Thus, the SU(3) Young tableau that represents the flavor state must be identical to the SU(2) tableau representing the spin state. In particular, the flavor tableau for a spin- $\frac{1}{2}$ nucleon must take the form of the $J=\frac{1}{2}$ tableau shown in figure 4.1. According to the rules of Young tableau, the tensors corresponding to this tableau are traceless, symmetric tensors with one upper index and $n$ lower indices. Thus, we are led to the conclusion that the $\mathrm{SU}(3)$ representation containing the nucleon should be chosen to be the $(1, n)$ representation. It is easy to show that this is in fact the only representation in the soliton hilbert space which contains states with strangeness zero and isospin 1/2. (For a more extensive discussion of this issue, see the article by Kaplan and Klebanov [22].)

Having decided upon a representation, the next step is to evaluate the ClebschGordan coefficients appearing in equation (4.8). This is done in reference [22]; I will simply quote the necessary results here:

$$
\begin{aligned}
& \left(\begin{array}{cc|c}
\mathbf{R} & \mathbf{8} & \mathbf{R} \\
P & \pi^{0} & P
\end{array}\right)_{F}=\frac{\sqrt{3}}{N+3} \\
& \left(\begin{array}{ll|l}
\mathbf{R} & \mathbf{8} & \mathbf{R} \\
P & \eta & P
\end{array}\right)_{F}=\frac{N}{N+3} \\
& \left(\begin{array}{cc|c}
\mathbf{R} & \mathbf{8} & \mathbf{R} \\
P & \pi^{0} & P
\end{array}\right)_{D}=\frac{1}{\sqrt{3}} \sqrt{\frac{N-1}{N+7}} \frac{N+6}{N+3} \\
& \left(\begin{array}{ll|l}
\mathbf{R} & \mathbf{8} & \mathbf{R} \\
P & \eta & P
\end{array}\right)_{D}=-\frac{3}{N+3} \sqrt{\frac{N-1}{N+7}}
\end{aligned}
$$

For convenience, I have used $\mathbf{R} \equiv(1, n)$. The subscripts $F$ and $D$ refer to the two $(1, n)$ representations contained in the direct product $(1, n) \times 8$. The labels $\pi^{0}$ and $\eta$ appearing 
in the coefficients denote $\mathrm{SU}(3)$ octet states with the isospin and hypercharge quantum numbers of these particles. For example, $\pi^{0}$ refers to a state with $Y=0, I=1$, and $I_{3}=0$. Putting everything together, we obtain:

$$
\begin{aligned}
& g_{T}^{3}=k \frac{(N-1)(N+6)^{2}+9(N+7)}{3(N+7)(N+3)^{2}} \rightarrow \frac{k}{3} \\
& g_{T}^{8}=k \frac{2 \sqrt{3}}{(N+3)(N+7)} \rightarrow \frac{2 \sqrt{3} k}{N^{2}}
\end{aligned}
$$

As previously discussed, we do not expect the Skyrme model to be reliable beyond leading order in the $1 / N_{c}$ expansion. Therefore, we should keep only the leading terms in $1 / N_{c}$ in these equations.

Apparently, the ratio $g_{T}^{8} / g_{T}^{3}$ vanishes in the large- $N_{c}$ limit, although we will see that it is expected to vanish linearly in $1 / N_{c}$, rather than quadratically. This statement applies to the proton tensor charges, but $\mathrm{SU}(3)$ symmetry enables us to extend it to a statement about all baryon matrix elements of the tensor charge operators. Briefly, the Wigner-Eckart theorem tells us that since there are two octet representations in the direct product of two octets, we can write all baryon matrix elements of the octet tensor charge operators in terms of two invariant matrix elements, and appropriate ClebschGordan coefficients. Let $|A\rangle$ and $|B\rangle$ be octet states, represented in the usual way by traceless tensors $A_{j}^{i}$ and $B_{j}^{i}\left(i . e .,|A\rangle=A_{j}^{i}\left|{ }_{i}^{j}\right\rangle\right)$. Let $M$ be an octet operator, also represented by a traceless tensor, $M_{j}^{i}$. The Wigner-Eckart theorem tells us that we can write the matrix element $\langle A|M| B\rangle$ as follows:

$$
\langle A|M| B\rangle=F \operatorname{Tr}(\bar{A}[M, B])+D \operatorname{Tr}(\bar{A}\{M, B\})
$$


This parameterization is valid for any choice of $|A\rangle,|B\rangle$, and $M$, provided that we restrict each state or operator to a single octet. According to this definition,

$$
\begin{aligned}
g_{T}^{3} & =F \operatorname{Tr}\left(\bar{P}\left[\lambda_{3}, P\right]\right)+D \operatorname{Tr}\left(\bar{P}\left\{\lambda_{3}, P\right\}\right) \\
& =F+D \\
g_{T}^{8} & =F \operatorname{Tr}\left(\bar{P}\left[\lambda_{8}, P\right]\right)+D \operatorname{Tr}\left(\bar{P}\left\{\lambda_{8}, P\right\}\right) \\
& =\sqrt{3} F-\frac{1}{\sqrt{3}} D
\end{aligned}
$$

Solving for $F / D$ and using the fact that $g_{T}^{8} / g_{T}^{3}$ vanishes in the large- $N_{c}$ limit, we obtain

$$
\frac{F}{D}=\frac{1+\sqrt{3} g_{T}^{8} / g_{T}^{3}}{3-\sqrt{3} g_{T}^{8} / g_{T}^{3}} \rightarrow \frac{1}{3}
$$

This prediction is independent of the renormalization scale, since $g_{T}^{3}$ and $g_{T}^{8}$ have the same anomalous dimension.

The results thus far can be summarized by saying that in the large- $N_{c}$ limit, the Skyrme model predicts that $g_{T}^{0} / g_{T}^{3}$ and $g_{T}^{8} / g_{T}^{3}$ vanish. For purposes of discussing corrections to these results, it will be useful to translate them into statements about the individual quark components, $\delta u, \delta d$, and $\delta s$. (This can be done unambiguously, since $g_{T}^{0}, g_{T}^{3}$, and $g_{T}^{8}$ all have the same anomalous dimension.) From equation, we obtain

$$
\begin{aligned}
\delta u & =\frac{1}{4} g_{T}^{3}+\frac{1}{4 \sqrt{3}} g_{T}^{8}+\frac{\sqrt{2}}{4 \sqrt{3}} g_{T}^{0} \\
\delta d & =\frac{-1}{4} g_{T}^{3}+\frac{1}{4 \sqrt{3}} g_{T}^{8}+\frac{\sqrt{2}}{4 \sqrt{3}} g_{T}^{0} \\
\delta s & =\frac{4}{\sqrt{3}} g_{T}^{8}+\sqrt{\frac{2}{3}} g_{T}^{0} .
\end{aligned}
$$

Thus, in the large- $N_{c}$ limit, $\delta d / \delta u \rightarrow-1$, and $\delta s / \delta u \rightarrow 0$. These predictions are in agreement with the predictions of the large- $N_{c} \mathrm{NQM}$, equation (4.6), as expected. 
Our results are derived from equation (3.24), which neglects the contributions of meson operators involving time-derivatives. As discussed previously, the contributions of such operators are expected to be suppressed by one or more factors of $1 / N_{c}$. This statement requires some interpretation. The leading order predictions for $\delta u$ and $\delta d$ are such that $\left|\delta u_{0}+\delta d_{0}\right| \ll\left|\delta u_{0}-\delta d_{0}\right|$. (The subscript 'zero' has been used to denote quantities calculated at leading order in the $1 / N_{c}$ expansion.) It is awkward to discuss corrections to a quantity which is small by virtue of a cancellation among its components. This is the position in which we find ourselves, however, when we consider corrections to $g_{T}^{8}$ and $g_{T}^{0}$. We can avoid some of these problems by considering, instead, the individual quark components, $\delta u, \delta d$, and $\delta s$. The quark components $\delta u_{0}$ and $\delta d_{0}$ do not appear to be suppressed by cancellations at leading order, so we can safely assume that corrections to these quantities are suppressed by $1 / N_{c}$ :

$$
\begin{aligned}
& \delta u=\delta u_{0}\left[1+O\left(\frac{1}{N_{c}}\right)\right] \\
& \delta d=\delta d_{0}\left[1+O\left(\frac{1}{N_{c}}\right)\right]
\end{aligned}
$$

There is no reason to assume that corrections to $\delta s_{0}$ will be small in comparison to the corrections to $\delta u_{0}$, so I will assume that they are of comparable size (i.e., the ratio of the corrections to these quantities is of order unity in the large- $N_{c}$ limit). We then obtain

$$
\frac{\delta s}{\delta u}=O\left(\frac{1}{N_{c}}\right)
$$

In terms of $g_{T}^{3}, g_{T}^{8}$, and $g_{T}^{0}$, these statements translate to

$$
\begin{aligned}
& \frac{g_{T}^{8}}{g_{T}^{3}}=O\left(\frac{1}{N_{c}}\right) \\
& \frac{g_{T}^{0}}{g_{T}^{3}}=O\left(\frac{1}{N_{c}}\right) .
\end{aligned}
$$


Finally, the prediction for the $F / D$ ratio becomes

$$
\frac{F}{D}=\frac{1}{3}+O\left(\frac{1}{N_{c}}\right) .
$$

We now consider the axial charges. For a nucleon at rest, only the space-components of the axial-vector current operator yield non-vanishing matrix elements. If we denote these by $A_{i}^{a}$, then the $A_{i}^{a}$ transform in exactly the same way as the $O_{i}^{a}$ under $\mathrm{SU}(3)$ $\times \mathrm{SU}(2)$. Thus, the analysis outlined above also applies to the axial charges, and we draw the same conclusions as before: The ratio $g_{A}^{8} / g_{A}^{3}$ is of order $1 / N_{c}$, and $F / D=$ $1 / 3+O\left(1 / N_{c}\right) ;$ the ratio $g_{A}^{0} / g_{A}^{3}$ vanishes, although it is actually expected to vanish like $1 / N_{c}^{2}$, rather than $1 / N_{c}$. (This last result does not contradict the arguments given above, which are only meant to place crude limits on the large- $N_{c}$ behavior.)

The results for the axial charges differ from previously published Skyrme model results. The commonly quoted prediction for the $F / D$ ratio is $5 / 9$, which results from choosing $\mathbf{R}=\mathbf{8}$ in equation (4.8) (see, for example, reference [23]). This amounts to setting $N_{c}=3$, and keeping terms of all orders in $1 / N_{c}$ in equation (4.11). This is inconsistent with the derivation of these equations, which neglected all but the leading terms in $1 / N_{c}$. Therefore, we should perhaps regard as fortuitous the good agreement between the experimental result $(F / D=0.58 \pm 0.05[24])$ and the 'prediction' of $5 / 9$. The proper prediction, $F / D=1 / 3+O\left(1 / N_{c}\right)$, clearly is not very precise for the realistic case of $N_{c}=3$, although it is at least consistent with the experimental result. In the case of the tensor charges, where no experimental information is available, it would seem that the only reasonable prediction we can make is $F / D=1 / 3+O\left(1 / N_{c}\right)$.

The commonly quoted prediction (i.e., $F / D=5 / 9$ ) has led to at least one other inconsistent prediction. As we have seen, $g_{A}^{0} / g_{A}^{3}$ is expected to vanish in the large- $N_{c}$ 
limit. If we take $F / D=5 / 9$, this leads to the prediction that $\Delta s / \Delta u$ is of order unity in the large- $N_{c}$ limit [25]. Since $\Delta u$ is of order $N_{c}$ [11] in the Skyrme model, we are led to the surprising (and inconsistent) result that the contribution of strange quarks to the spin of the proton is of order $N_{c}$. In fact, as we have seen, a consistent treatment of the large- $N_{c}$ limit leads to the prediction that $\Delta s / \Delta u$ is of order $1 / N_{c}$, implying that $\Delta s=O(1)$. This is consistent with the suppression of the $q \bar{q}$ sea that is expected on general grounds in large- $N_{c}$ QCD [16].

The experimental result for the ratio $g_{A}^{0} / g_{A}^{3}$ is in fact small. Using the experimental values $g_{A}^{0}=0.098 \pm 0.076 \pm 0.113$ and $g_{A}^{3}=1.254 \pm 0.006[26]$, we obtain $g_{A}^{0} / g_{A}^{3}=$ $0.078 \pm 0.109$. Unfortunately, this result does not offer a useful standard against which to compare the prediction $g_{T}^{0} / g_{T}^{3}=O\left(\frac{1}{N_{c}}\right)$, since $g_{A}^{0} / g_{A}^{3}$ is expected to be of order $1 / N_{c}^{2}$.

Finally, we discuss the effects of SU(3) symmetry violation on the above results. The role of the strange quark in the nucleon is poorly understood in general. In the particular case of the tensor charges, it does not seem possible to say anything rigorous about the effects of a symmetry-breaking mass term for the strange quark. Within the framework of the Skyrme model, for example, we might approach this problem from the point of view of dimensional analysis. Unfortunately, dimensional analysis appears to be completely unreliable for $\mathrm{SU}(3)$ symmetry-breaking effects in the Skyrmion, since the dimensionful parameter that sets the scale for symmetry-breaking effects is of order 250 $\mathrm{MeV}$ (i.e., less than the kaon mass, a typical measure of symmetry violation in the chiral lagrangian) [22]. We simply cannot rule out the possibility of large corrections stemming from $\mathrm{SU}(3)$ violation. For what it is worth, we point out that $\mathrm{SU}(3)$ appears to be a very good symmetry in the case of the axial charges, although again, the reasons for this are not well understood. 


\section{SUMMARY}

We consider the nucleon tensor charges in the Skyrme model. We show that in the large- $N_{c}, \mathrm{SU}(3)$-symmetric limit, the model predicts that $g_{T}^{8} / g_{T}^{3}$ is of order $1 / N_{c}$. This is equivalent to the prediction $F / D=1 / 3+O\left(\frac{1}{N_{c}}\right)$, which, unfortunately, is not very precise for the realistic case of $N_{c}=3$. These predictions are identical to the Skyrme model predictions for the octet axial charges. The model also predicts that $g_{T}^{0} / g_{T}^{3}$ is of order $1 / N_{c}$. All of these predictions are in agreement with the predictions of the NQM. Unlike the NQM, the Skyrme model does not readily indicate the large- $N_{c}$ behavior of, say, $g_{T}^{3}$, although it is not inconsistent to assume that $g_{T}^{3}$ is of order $N_{c}$ in the Skyrme model. We point out that the commonly quoted prediction for the axial charge $F / D$ ratio (i.e., 5/9) is based on an inconsistent treatment of the large- $N_{c}$ limit. A consistent treatment yields the prediction quoted above, and shows that the strange quark contribution to the nucleon spin is of order unity in the large- $N_{c}$ Skyrme model. 


\section{APPENDIX: Anomalous Dimension of Tensor Charges}

The tensor charge operators, $\Sigma_{\mu \nu}^{a}$, will be defined as follows:

$$
\Sigma_{\mu \nu}^{a}=\bar{\Psi} \lambda^{a} \sigma_{\mu \nu} i \gamma^{5} \Psi .
$$

As it stands, $\Sigma_{\mu \nu}^{a}$ has divergent matrix elements. Therefore, we define the renormalized tensor charge operator, $\Sigma_{\mu \nu(R)}^{a}$ :

$$
\Sigma_{\mu \nu(R)}^{a}=\frac{1}{Z_{\Sigma}} \bar{\Psi} \lambda^{a} \sigma_{\mu \nu} i \gamma^{5} \Psi .
$$

The anomalous dimension of $\Sigma_{\mu \nu}^{a}$ is defined in terms of the renormalization constant, $Z_{\Sigma}$, as follows:

$$
\gamma=\mu \frac{\partial \ln Z_{\Sigma}}{\partial \mu}
$$

where $\mu$ is the renormalization mass scale.

To renormalize $\Sigma_{\mu \nu}^{a}$ to one-loop order, we consider the various one-loop graphs that might be divergent. There are only three potentially divergent graphs; see figure A.1.
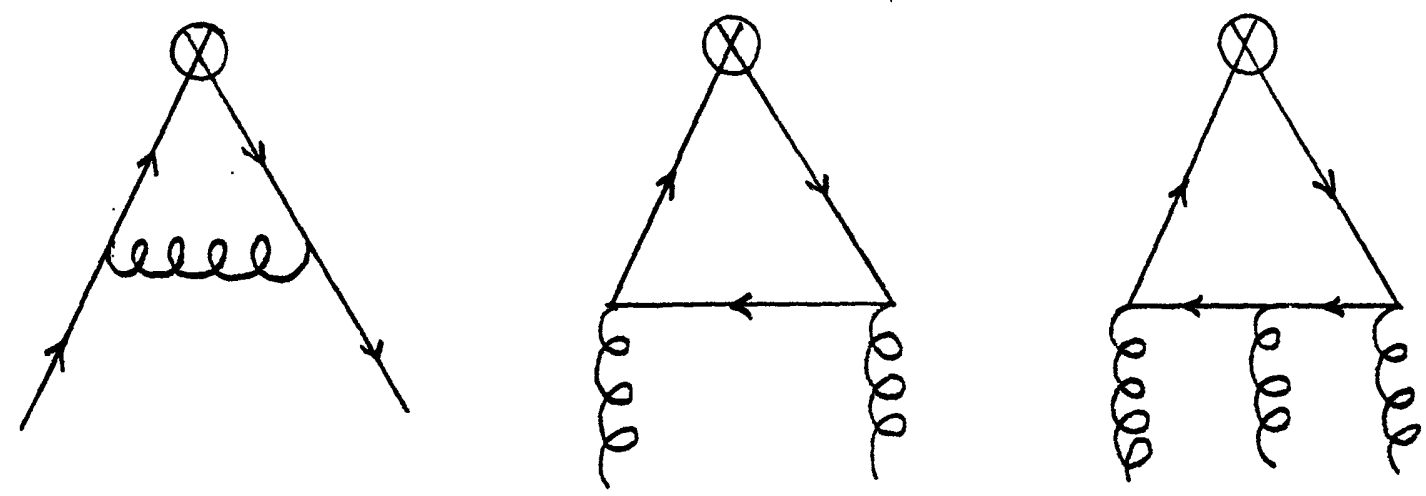

Fig. A.1: One-loop graphs for renormalization of tensor charge operator. 
Surprisingly, all three graphs are finite. It is trivial to prove this, so I will not bother to do so. Note that since the graphs involving external gluons are finite, $\Sigma_{\mu \nu}^{a}$ does not mix with gluon operators.

There is still one source of divergence to account for, namely, the divergence of the quark field operators themselves. Thus far, we have implicitly ignored two graphs; see figure A.2. These two graphs have nothing to do with $\Sigma_{\mu \nu}^{a}$, but serve only to renormalize
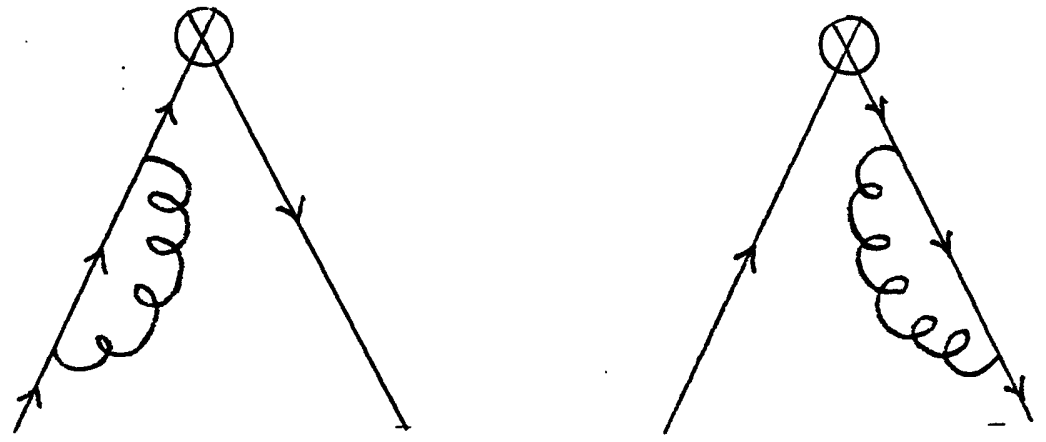

Fig. A.2: Quark self-energy graphs.

the quark field operators. They are also entirely responsible for the one-loop divergence of $\Sigma_{\mu \nu}^{a}$. Thus, the bare and renormalized versions of the tensor charge operator are related as follows:

$$
\Sigma_{\mu \nu}^{a}=Z_{\psi} \bar{\Psi}_{(R)} \lambda^{a} \sigma_{\mu \nu} i \gamma^{5} \Psi_{(R)}=Z_{\psi} \Sigma_{\mu \nu(R)}^{a},
$$

where $\Psi_{(R)}$ is the renormalized quark operator. We conclude that $Z_{\Sigma}=Z_{\psi}$. In QCD, using dimensional regularization and minimal subtraction, $Z_{\psi}$ is given by:

$$
Z_{\psi}=1-\frac{g^{2}}{6 \pi^{2} \epsilon}
$$


Thus, for the tensor charge anomalous dimension, we obtain

$$
\begin{aligned}
\gamma & =\mu \frac{\partial \ln Z_{\psi}}{\partial \mu} \\
& =\mu \frac{\partial g}{\partial \mu} \frac{\partial \ln Z_{\psi}}{\partial g} \\
& =\left(\frac{-\epsilon g}{2}+\beta(g)\right)\left(\frac{-g}{3 \pi^{2} \epsilon}\right) \\
& =\frac{g^{2}}{6 \pi^{2}} .
\end{aligned}
$$

The QCD fine structure constant is defined as $\alpha_{s}=g^{2} / 4 \pi$, so we obtain the result $\gamma=2 \alpha_{s} / 3 \pi$. (The anomalous dimensions for the complete family of twist-two operators associated with $h_{1}(x)$ have been calculated at one-loop order by Kodaira et al. [27], and by Artru and Mekhfi [28].) 


\section{BIBLIOGRAPHY}

[1] R. L. Jaffe and Xiangdong Ji, Nucl. Phys. B375 (1992) 527.

[2] For a review and references, see A. Manohar, UCSD/PTH 92-10 (1992).

[3] For a review and references, see reference 1.

[4] J. Collins, Penn State Preprint, 1990

[5] J. Ralston and D. E. Soper, Nucl. Phys. B152 (1979) 109.

[6] See, for example, C. H. LLewellyn Smith, Proceedings of the 1984 PANIC Conference, Heidelberg.

[7] D. J. Gross, Methods in Field Theory, Proc. of 1975 Les Houches Summer School, ed., R. Balian and J. Zinn-Justin, North-Holland, Amsterdam.

[8] See, for example, R.K. Ellis, H. Georgi, M. Machacek, H.D. Politzer, and G.G. Ross, Nucl. Phys. B152 (1979) 285.

[9] R.L. Jaffe, Deep-Inelastic Scattering with Applications to Nuclear Targets, in Relativistic Dynamics and Quark Nuclear Physics, eds., M.B. Johnson and A. Picklesimer (1985).

[10] R.L. Jaffe, Nucl. Phys. B229 (1983) 205.

[11] G. Adkins, C. Nappi, and E. Witten, Nucl. Phys. B228 (1983) 552.

[12] E. Guadagnini Nucl. Phys. B236 (1984) 35.

[13] See, for example, S. Coleman, Aspects of Symmetry, Cambridge University Press (1985).

[14] J. Goldstone and F. Wilczek, Phys. Rev. Lett. 47 (1981) 986. 
[15] A. Manohar and H.Georgi, Nucl. Phys. B234 (1984) 189.

[16] E. Witten, Nucl. Phys. B160 (1979) 57.

[17] J. Wess and B. Zumino, Phys. Lett B37 (1971) 95.

[18] E. Witten, Nucl. Phys. B223 (1983) 422.

[19] A. Manohar, Nucl. Phys. B248 (1984) 19.

[20] G. Karl and J.E. Paton, Phys. Rev. D30 (1984) 238.

[21] See, for example, H. Georgi, Lie Algebras in Particle Physics, Benjamim Cummings (1982).

[22] D. Kaplan and I. Klebanov, Nucl. Phys. B335 (1990) 45.

[23] J. Bijnens, H. Sonoda, and M. Wise, Phys. Lett. B140 (1984) 421.

[24] R.L. Jaffe and A. Manohar, Nucl. Phys. B337 (1990) 509.

[25] S. Brodsky, J. Ellis, and M. Karliner, Phys. Lett. B206 (1988) 309.

[26] J. Ashman et al., Nucl. Phys. B337 (1990) 509.

[27] J. Kodaira, S. Matsuda, K. Sasaki, and T. Uematsu, Nucl. Phys. B159 (1979) 99.

[28] X. Artru and M. Mekhfi, Z. Physik C45 (1990) 669.

[29] See, for example, J.J. Sakurai, Modern Quantum Mechanics, Addison-Wesley (1985). 